\title{
Polynomial-time probabilistic controllability analysis of discrete- time piecewise affine systems
}

$\operatorname{AUTHOR}(S):$

Azuma, Sl; Imura, JI

\section{CITATION:}

Azuma, SI ...[et al]. Polynomial-time probabilistic controllability analysis of discrete-time piecewise affine systems. IEEE TRANSACTIONS ON AUTOMATIC CONTROL 2007, 52(11): 2029-2046

\section{ISSUE DATE:}

2007-11

URL:

http://hdl.handle.net/2433/50325

\section{RIGHT:}

(c)2007 IEEE. Personal use of this material is permitted. However, permission to reprint/republish this material for advertising or promotional purposes or for creating new collective works for resale or redistribution to servers or lists, or to reuse any copyrighted component of this work in other works must be obtained from the IEEE. 


\title{
Polynomial-Time Probabilistic Controllability Analysis of Discrete-Time Piecewise Affine Systems
}

\author{
Shun-Ichi Azuma, Member, IEEE, and Jun-Ichi Imura, Member, IEEE
}

\begin{abstract}
This paper proposes a probabilistic approach to the controllability analysis for discrete-time piecewise affine (PWA) systems. Three kinds of randomized algorithms, which are based on random sampling of the mode sequence and/or the initial state, for determining with a probabilistic accuracy if the system is controllable are presented: a positive one-sided error algorithm, a negative one-sided error algorithm, and a two-sided error algorithm. It is proven that these are polynomial-time algorithms with respect to several variables of the problem. It is also shown with some examples, for which it is hopeless to check the controllability in a deterministic way, that these algorithms are efficient.
\end{abstract}

Index Terms-Controllability, hybrid systems, piecewise affine (PWA) systems, randomized algorithms, reachability.

\section{INTRODUCTION}

I $\mathrm{N}$ the research field of hybrid systems, the controllability (reachability) problem is one of the fundamental and important research issues for developing the controller synthesis techniques; so since from the beginning stage to the current stage in the research of hybrid systems, various approaches to the problem have been extensively developed. In fact, for some classes of the hybrid systems such as periodic hybrid systems and switched linear systems, useful results on this topic have been already obtained (see, e.g., [1] and [2]). On the other hand, for piecewise affine (PWA) systems and mixed logical dynamical (MLD) systems, this kind of problem has been negatively solved in the sense that it is undecidable over infinite time [3], [4]. In addition, it has been shown in [5] that the PWA system is not always controllable (uncontrollable) even if the subsystem in every mode is controllable (uncontrollable) in the usual sense. Thus, it is known that the controllability analysis for this class of hybrid systems is a challenging research topic.

In spite of such theoretical limitations, several results have been obtained based on a deterministic approach. Bemporad et al. have discussed the controllability problem over finite time for the discrete-time PWA/MLD systems, where the problem is reduced into an mixed-integer linear programming problem [5]. In addition, they have proposed an algorithmic technique based on the computation of all system evolutions, in which the feasible discrete transitions and the reach sets are

Manuscript received August 27, 2004; revised July 7, 2006. Recommended by Associate Editor A. Bemporad.

S. Azuma is with the Graduate School of Informatics, Kyoto University, Kyoto 611-0011, Japan (e-mail: sazuma@i.kyoto-u.ac.jp).

J. Imura is with the Graduate School of Information Science and Engineering, Tokyo Institute of Technology, Tokyo 152-8552, Japan (e-mail: imura@cyb. mei.titech.ac.jp).

Digital Object Identifier 10.1109/TAC.2007.908308 derived by solving mixed-integer linear programming problems and projecting polyhedra, respectively [6], [7]. However, the mixed-integer linear programming problems are, in general, NP-hard, and the existing projection methods Vertex enumeration, Fourier-Motzkin elimination, and Block elimination require exponentially growing computation with respect to some of the problem variables. Recently, in the line of this approach, the multiparametric optimization technique has been proposed in [8]-[10]; however, this powerful method also has a large computational complexity. From the different points of view, the authors have derived in [11] an easily checkable sufficient condition for a new class of hybrid systems, i.e., continuous-time PWA systems with sampled-data switching (called the sampled-data PWA systems [12], [13]), to be controllable. However, since it is, in general, conservative, an impractical condition will be obtained for discrete-time PWA systems if the same idea is applied. Furthermore, similar algebraic approaches have been obtained for various types of hybrid systems [14]-[16].

In addition to the aforementioned contributions, an approach based on Hamilton-Jacobi (H-J) equations has been studied for developing the controller synthesis with reachability specification for continuous-time hybrid systems (e.g., [17]-[19]). This approach also causes computational hardness associated with solving nonsmooth $\mathrm{H}-\mathrm{J}$ equations. Thus, the approximation methods using level sets for solving the $\mathrm{H}-\mathrm{J}$ equations have been recently presented (see, e.g., [20]). Furthermore, in the computer science community, symbolic methods for computing the set of reachable state have been developed in [21] and [22], and various kinds of approximation approaches to the reachability (verification) problem have been extensively studied for improving the computational complexities (see, e.g., [23]-[25]). It should be noted here that all methods for these kinds of problems are based on a deterministic approach, in which no randomness is used for solving the problems.

On the other side, an alternative, possible approach to approximately solve the controllability and reachability problems in a practically short time is to use a probabilistic method. For example, in a production system design of a chemical plant, it is important to verify with a sufficiently high probabilistic accuracy that there exists a control input driving the state of the system from an emergency state caused by some disturbance to a safe state. However, no probabilistic approach to the controllability and reachability problems of hybrid systems has been developed so far, although the probabilistic gradient-based method for robust control [26] has been recently extended to the stability and stabilization problems of quantized sampled-data systems and switched linear systems [27], [28], and some probabilistic 
approaches to analyze properties of complex systems (hybrid systems) have been presented in [29] and [30].

This paper proposes a polynomial-time approach based on a probabilistic method [31]-[33] to the controllability analysis of discrete-time PWA systems. The main contributions of this paper are as follows.

First, three randomized algorithms for approximately solving the controllability problem with a probabilistic accuracy are proposed: a positive one-sided error algorithm (algorithm P) based on sampling the mode sequence, a negative one-sided error algorithm (algorithm $\mathrm{N}$ ) based on sampling the initial state, and a two-sided error algorithm (algorithm T) based on sampling both the mode sequence and the initial state.

Next, it is proven that they are polynomial-time algorithms with respect to several variables of the problem, and that they are complementary to each other from the viewpoints of the decision results and computational complexities. More specifically, algorithm $\mathrm{P}$ is a polynomial-time algorithm with the control time period and the other variables except for the dimension of the state, which can determine if the system is controllable or is uncontrollable with a probabilistic accuracy. In contrast, algorithm $\mathrm{N}$ is a polynomial-time algorithm with the dimension of the state and the other variables except for the control time period, which can determine if the system is controllable with a probabilistic accuracy or is uncontrollable. Further, algorithm $\mathrm{T}$ is a polynomial-time algorithm with all the variables, which can determine if the system is either controllable or uncontrollable with a probabilistic accuracy.

Finally, a polynomial-time algorithm for projecting polyhedra is developed, which is a crucial technique for proving that algorithm $\mathrm{P}$ is a polynomial-time algorithm with the control time period. This algorithm can be also applied to the general case such as polyhedra that are neither open nor closed.

In Section II, the systems and the controllability notion to be studied are described, and then a necessary and sufficient condition for the system to be controllable is derived. Based on this, in Section III, three probabilistic algorithms are proposed, and it is proven that they are polynomial-time algorithms. In addition, several techniques to more efficiently execute the algorithms are proposed in Section IV. Finally, Section V gives numerical examples and Section VI concludes this paper.

Notation: Let $\mathcal{R}, \mathcal{N}$, and $\mathcal{N}_{+}$denote the real number field, the set of nonnegative integers, and the set of positive integers, respectively. We denote by $0_{n \times m}, \boldsymbol{I}_{n}$, and $\mathbf{1}_{n}$ (or, for simplicity of notation, $0, \boldsymbol{I}$, and $\mathbf{1}$ ) the $n \times m$ zero matrix, the $n \times n$ identity matrix, and the $n \times 1$ vector whose all elements are one, respectively, by $\lfloor a\rfloor$ and $\lceil a\rceil$ the floor and the ceil of the positive number $a$, and by $\left(\begin{array}{c}m \\ n\end{array}\right)$ the binomial coefficient of the nonnegative integers $m$ and $n(m \geq n, m \geq 1)$. We use $\operatorname{span}(V)$ to express the vector space spanned by the column vectors of the matrix $V$, and use $x_{(i)}$ to express the $i$ th element of the vector $x$. The vector inequality $x_{1} \leq(<) x_{2}$ expresses that each element of $x_{1}-x_{2}$ is nonpositive (negative). For the sets $\mathcal{X}_{1}$ and $\mathcal{X}_{2}$, $\mathcal{X}_{1}-\mathcal{X}_{2}$ expresses the difference set and $\mathcal{X}_{1} \subseteq \mathcal{X}_{2}\left(\mathcal{X}_{1} \subset \mathcal{X}_{2}\right)$ expresses that $\mathcal{X}_{1}$ is a subset (a strict subset) of $\mathcal{X}_{2}$. We denote by $\operatorname{vol}(\mathcal{X})$ the measure of the measurable set $\mathcal{X}$ of the vector $x$ as $\operatorname{vol}(\mathcal{X}):=\int_{\mathcal{X}} d x$ and by $\operatorname{card}(\mathcal{I})$ the cardinality of the finite set $\mathcal{I}$. Let also hull $(\mathcal{X})$ and $\operatorname{ri}(\mathcal{X})$ denote the convex hull and the relative interior of the set $\mathcal{X}$, respectively. The set $\mathcal{S}$ given as the form $\mathcal{S}:=\left\{x \in \mathcal{R}^{n} \mid A x+b \leq 0, C x+d<0\right\}$ is called here the polyhedron, where $A$ and $C$ are some matrices and $b$ and $d$ are some column vectors. Finally, for the bounded and closed polyhedron $\mathcal{P}$, let vert $(\mathcal{P})$ and $\operatorname{dim}(\mathcal{P})$ be the set of all vertices and the dimension (defined as the dimension of the affine hull of $\mathcal{P}$ ), respectively. In addition, $\mathcal{P} \cap\left\{x \in \mathcal{R}^{n} \mid G x+g=0\right\}$ is called a face, where $G$ and $g$ are some row vector and scalar satisfying $G x^{\prime}+g \leq 0$ for every $x^{\prime} \in \mathcal{P}$. Note that $\mathcal{P}$ itself is one of the faces of $\mathcal{P}$.

\section{Controllability of Discrete-Time PWA Systems}

In this section, some preliminary results are given for the probabilistic controllability analysis proposed in Section III.

\section{A. Discrete-Time PWA Systems and Controllability Notion}

This paper considers the discrete-time PWA system $\Sigma$

$$
\begin{aligned}
& x(k+1)=A_{I(k)} x(k)+B_{I(k)} u(k)+a_{I(k)} \\
& I(k+1)=I_{+}, \quad \text { if } x(k+1) \in \mathcal{S}_{I_{+}}
\end{aligned}
$$

where $x \in \mathcal{R}^{n}$ is the state (the continuous-valued state), $I \in \mathcal{I}$ is the mode, $\mathcal{I}:=\{0,1, \ldots, M-1\}$ is the set on which the mode takes values, $M \in \mathcal{N}_{+}$is the number of the mode values, $u \in \mathcal{R}^{m}$ is the control input, $A_{I} \in \mathcal{R}^{n \times n}, B_{I} \in \mathcal{R}^{n \times m}$, and $a_{I} \in \mathcal{R}^{n}$ are constant matrices for mode $I, k \in \mathcal{N}$ is the sampling time, and $I_{+} \in \mathcal{I}$ is the value of the mode at the $(k+1)$ th discrete time. In addition, $\mathcal{S}_{I}$ denotes the subregion of the state assigned to $I \in \mathcal{I}$, given by the polyhedron

$$
\mathcal{S}_{I}:=\left\{x \in \mathcal{R}^{n} \mid C_{I} x+d_{I} \leq 0, \hat{C}_{I} x+\hat{d}_{I}<0\right\}
$$

where $C_{I} \in \mathcal{R}^{p_{I} \times n}, d_{I} \in \mathcal{R}^{p_{I}}, \hat{C}_{I} \in \mathcal{R}^{\hat{p}_{I} \times n}$, and $\hat{d}_{I} \in$ $\mathcal{R}^{\hat{p}_{I}}$. For this subregion, it is assumed that $\bigcup_{I \in \mathcal{I}} \mathcal{S}_{I}=\mathcal{R}^{n}$ and $\mathcal{S}_{I} \cap \mathcal{S}_{J}=\emptyset$ for every $I, J \in \mathcal{I}$ such that $I \neq J$. This assumption guarantees that $I$ is uniquely determined for each $x$, in other words, $\Sigma$ is well posed (for every $x(0) \in$ $\mathcal{R}^{n}$ and $u(k) \in \mathcal{R}^{m}(k=0,1, \ldots)$, a unique solution $x(k)$ exists for all $k \in\{0,1, \ldots\})$. For simplicity of notation, we often use $x(0)=x_{0}\left(x_{0} \in \mathcal{R}^{n}\right)$ as the initial state instead of $(I(0), x(0))=\left(I_{0}, x_{0}\right) \in\left\{(I, x) \in \mathcal{I} \times \mathcal{R}^{n} \mid x \in \mathcal{S}_{I}\right\}$, since by assumption, the value of the initial mode $I_{0} \in \mathcal{I}$ is uniquely determined by each $x_{0} \in \mathcal{R}^{n}$.

For the system $\Sigma$, the notion of controllability is defined as follows.

Definition 1: For the system $\Sigma$, suppose that the final time $T \in \mathcal{N}_{+}$and the direct product set $\mathcal{X} \subseteq \mathcal{R}^{n(T+1)}$ of the state sets $\mathcal{X}_{0}, \mathcal{X}_{1}, \ldots, \mathcal{X}_{T} \subseteq \mathcal{R}^{n}$, i.e., $\mathcal{X}:=\mathcal{X}_{0} \times \mathcal{X}_{1} \times \cdots \times$ $\mathcal{X}_{T}$, are given. Then, $\Sigma$ is said to be $(\boldsymbol{T}, \mathcal{X})$-controllable if for each $x_{0} \in \mathcal{X}_{0}$, there exists an input vector sequence $U:=$ $\{u(0), u(1), \ldots, u(T-1)\} \in \mathcal{R}^{m T}$ satisfying the following:

C1) $x(k) \in \mathcal{X}_{k}(k=1,2, \ldots, T)$

under the initial state $x(0)=x_{0}$. Otherwise, $\Sigma$ is said to be $(\boldsymbol{T}, \mathcal{X})$-uncontrollable.

Note that, in Definition 1 , the sets $\mathcal{X}_{0}, \mathcal{X}_{k}(k=$ $1,2, \ldots, T-1)$, and $\mathcal{X}_{T}$ of the initial, intermediate, and 
final state, respectively, and the final time $T$ are explicitly specified. The constraints on $x(k)(k=0,1, \ldots, T)$ are not special but a more generalized version of controllability notion than the usual ones; e.g., the special case of $\mathcal{X}_{0}:=\mathcal{R}^{n}$, $\mathcal{X}_{k}:=\mathcal{R}^{n}(k=1,2, \ldots, T-1)$, and $\mathcal{X}_{T}:=\{0\}$ corresponds to the usual notion over finite time (e.g., [5]).

In addition, this kind of definition is useful for considering various practical applications. For example, by the controllability analysis, it can be checked whether the finite-time optimal control (model predictive control) problem with the intermediate and/or final states constrained is feasible. In fact, if it is proven that some system $\Sigma$ is $(T, \mathcal{X})$-controllable for $\mathcal{X}:=[-10,10]^{n T} \times\{0\}$, it is guaranteed that the finite-time optimal control problem of the system with the state constraints $x(k) \in[-10,10]^{n}(k=1,2, \ldots, T-1)$, and the fixed final state $x(T)=0$ is feasible for any initial state $x(0)=x_{0} \in$ $[-10,10]^{n}$, which thus implies that the corresponding receding horizon policy on the prediction interval $[k, k+T]$ can be applied to this system. Such a feasibility often has to be checked in the process control design for a chemical plant. In addition, the definition allows us to consider the mode constraint such as $I(1) \in\{0,1\}$ and $I(2)=2$, by setting $\mathcal{X}_{k}\left(\right.$ as $\mathcal{X}_{1}:=\mathcal{S}_{0} \cup \mathcal{S}_{1}$ and $\mathcal{X}_{2}:=\mathcal{S}_{2}$ ).

In the following sections, we suppose that $T \in\{2,3, \ldots\}$ for simplicity of discussion, since we have to use different symbols for the cases $T=1$ and $T \in\{2,3, \ldots\}$ to characterize the $(T, \mathcal{X})$-controllability (although the techniques and the obtained results are the same for both cases). Furthermore, for simplicity of discussion, this paper omits the case that $\Sigma$ has 1) the constraints on the control input and/or 2) the discrete transition depending on the control input. Even to such a case, all the results derived in the following sections can be applied with some modifications (e.g., see [13]).

\section{B. Controllability Condition}

First, let $\mathcal{X}_{i m}^{T-1}$ be the direct product set of the $T-1$ sets $\mathcal{X}_{1}, \mathcal{X}_{2}, \ldots, \mathcal{X}_{T-1}$ of the intermediate state, i.e., $\mathcal{X}_{i m}^{T-1}:=$ $\mathcal{X}_{1} \times \mathcal{X}_{2} \times \cdots \times \mathcal{X}_{T-1}$ (note $\left.\mathcal{X}=\mathcal{X}_{0} \times \mathcal{X}_{i m}^{T-1} \times \mathcal{X}_{T}\right)$. We denote by $(\mathbb{D}, \mathbb{X})$ a sequence of the intermediate mode and state pairs $\left(I_{k}, x_{k}\right) \in\left\{(I, x) \in \mathcal{I} \times \mathcal{R}^{n} \mid x \in \mathcal{S}_{I}\right\}(k=1,2, \ldots, T-1)$, where $\mathbb{0}:=\left[\begin{array}{llll}I_{1} & I_{2} & \cdots & I_{T-1}\end{array}\right]^{\top} \in \mathcal{I}^{T-1}$ and $\mathbb{X}:=$ $\left[\begin{array}{llll}x_{1}^{\top} & x_{2}^{\top} & \cdots & x_{T-1}^{\top}\end{array}\right]^{\top} \in \mathcal{S}_{\mathbb{0}}$ for $\mathcal{S}_{\mathbb{1}}:=\mathcal{S}_{I_{1}} \times \mathcal{S}_{I_{2}} \times \cdots \times \mathcal{S}_{I_{T-1}}$ (1 is called the mode sequence). Then, in terms of the intermediate state $(\mathbb{D}, \mathbb{X})$, we will characterize a condition for the system to have $U \in \mathcal{R}^{m T}$ satisfying C1) for the initial state $(I(0), x(0))=\left(I_{0}, x_{0}\right)$.

Lemma 1: For the system $\Sigma$, suppose that $T \in\{2,3, \ldots\}$, $\left(I_{0}, x_{0}\right) \in\left\{(I, x) \in \mathcal{I} \times \mathcal{R}^{n} \mid x \in \mathcal{S}_{I}\right\}, \llbracket \in \mathcal{I}^{T-1}, \mathbb{X} \in \mathcal{S}_{\rrbracket}$, and $x_{T} \in \mathcal{R}^{n}$ are given. Then, the following statements are equivalent.

1) For the initial state $x(0)=x_{0}$ (i.e., $(I(0), x(0))=$ $\left(I_{0}, x_{0}\right)$ ), there exists an input vector sequence $U \in \mathcal{R}^{m T}$ satisfying $\left[\begin{array}{llll}I(1) & I(2) & \cdots & I(T-1)\end{array}\right]^{\top}=\mathbb{\rrbracket}$, $\left[\begin{array}{llll}x^{\top}(1) & x^{\top}(2) & \cdots & x^{\top}(T-1)\end{array}\right]^{\top}=\mathbb{X}$, and $x(T)=x_{T}$.

2) The following relation holds:

$$
E\left(I_{0}, x_{0}, \mathbb{}, \mathbb{X}, x_{T}\right)=0
$$

where

$$
E\left(I_{0}, x_{0}, \mathbb{\rrbracket}, \mathbb{X}, x_{T}\right):=E_{I_{0} \rrbracket}^{a}\left[\begin{array}{c}
x_{0} \\
\mathbb{X} \\
x_{T}
\end{array}\right]+E_{I_{0} \rrbracket}^{b}
$$

and $E_{I_{0} \rrbracket}^{a}$ and $E_{I_{0} \rrbracket}^{b}$ are given by

$$
\begin{aligned}
E_{I_{0} \rrbracket}^{a} & :=\left[\begin{array}{ccccc}
E_{I_{0}}^{1} & E_{I_{0}}^{2} & 0 & \cdots & 0 \\
0 & E_{I_{1}}^{1} & E_{I_{1}}^{2} & \ddots & \vdots \\
\vdots & \ddots & \ddots & \ddots & 0 \\
0 & \cdots & 0 & E_{I_{T-1}}^{1} & E_{I_{T-1}}^{2}
\end{array}\right] \\
E_{I_{0} \rrbracket}^{b} & :=\left[\begin{array}{c}
E_{I_{0}}^{3} \\
E_{I_{1}}^{3_{0}} \\
\vdots \\
E_{I_{T-1}}^{3}
\end{array}\right]
\end{aligned}
$$

and $E_{I_{k}}^{1}:=-\left(B_{I_{k}}^{\perp}\right)^{\top} A_{I_{k}}, E_{I_{k}}^{2}:=\left(B_{I_{k}}^{\perp}\right)^{\top}$, and $E_{I_{k}}^{3}:=-\left(B_{I_{k}}^{\perp}\right)^{\top} a_{I_{k}}$ for a matrix $B_{I}^{\perp} \in \mathcal{R}^{n \times m^{\perp}}$ satisfying $\operatorname{rank}\left[B_{I} B_{I}^{\perp}\right]=n$ and $\left(B_{I}^{\perp}\right)^{\top} B_{I}=0\left(m^{\perp} \in \mathcal{N}_{+}\right.$ is a positive integer).

Proof: For the system $x(k+1)=A_{I(k)} x(k)+B_{I(k)} u(k)$ $+a_{I(k)}$ with the state $(I(k), x(k))=\left(I_{k}, x_{k}\right)$, there exists a $u(k) \in \mathcal{R}^{m}$ satisfying $x(k+1)=x_{k+1}$ if and only if $x_{k+1}-A_{I_{k}} x_{k}-a_{I_{k}} \in \operatorname{span}\left(B_{I_{k}}\right)$. Then, $\operatorname{since} \operatorname{span}\left(B_{I}^{\perp}\right)$ expresses the orthogonal complement of $\operatorname{span}\left(B_{I}\right)$, the condition $x_{k+1}-A_{I_{k}} x_{k}-a_{I_{k}} \in \operatorname{span}\left(B_{I_{k}}\right)$ is equivalent to $\left(B_{I_{k}}^{\perp}\right)^{\top}\left(x_{k+1}-A_{I_{k}} x_{k}-a_{I_{k}}\right)=0$, which can be expressed as $E_{I_{k}}^{1} x_{k}+E_{I_{k}}^{2} x_{k+1}+E_{I_{k}}^{3}=0$. Thus, 1) holds if and only if $E_{I_{k}}^{1} x_{k}+E_{I_{k}}^{2} x_{k+1}+E_{I_{k}}^{3} \stackrel{I_{k}}{=} 0$ for every $k \in\{0,1, \ldots, T-1\}$, i.e., 2) holds.

From Lemma 1, it follows that for given $T \in\{2,3, \ldots\}$, $\left(I_{0}, x_{0}\right) \in\left\{(I, x) \in \mathcal{I} \times \mathcal{R}^{n} \mid x \in \mathcal{S}_{I}\right\}, \rrbracket \in \mathcal{I}^{T-1}, \mathcal{X}_{i m}^{T-1} \subseteq$ $\mathcal{R}^{n(T-1)}$, and $\mathcal{X}_{T} \subseteq \mathcal{R}^{n}$, there exists a $U \in \mathcal{R}^{m T}$ satisfying $[I(1) I(2) \cdots I(T-1)]^{\top}=\mathbb{\square}$ and $\left.C 1\right)$ under the initial state $(I(0), x(0))=\left(I_{0}, x_{0}\right)$ if and only if there exists an $\left[\mathbb{X}^{\top} x_{T}^{\top}\right]^{\top} \in\left(\mathcal{S}_{\mathbb{}} \cap \mathcal{X}_{i m}^{T-1}\right) \times \mathcal{X}_{T}$ satisfying (3), i.e.,

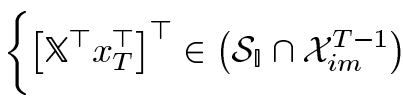

$$
\begin{aligned}
& \left.\times \mathcal{X}_{T} \mid E\left(I_{0}, x_{0}, \emptyset, \mathbb{X}, x_{T}\right)=0\right\} \neq \emptyset
\end{aligned}
$$

holds. Note that $\mathcal{S}_{\mathbb{}} \cap \mathcal{X}_{i m}^{T-1}=\left(\mathcal{S}_{I_{1}} \cap \mathcal{X}_{1}\right) \times\left(\mathcal{S}_{I_{2}} \cap \mathcal{X}_{2}\right) \times \cdots \times$ $\left(\mathcal{S}_{I_{T-1}} \cap \mathcal{X}_{T-1}\right)$. Then, for given $I_{0} \in \mathcal{I}$ and $\rrbracket \in \mathcal{I}^{T-1}$, we consider the set of $x_{0} \in \mathcal{S}_{I_{0}} \cap \mathcal{X}_{0}$ satisfying the aforementioned condition, that is

$$
\begin{aligned}
& \mathcal{X}_{c}^{I_{0} \rrbracket}(T, \mathcal{X}) \\
& :=\left\{x_{0} \in \mathcal{S}_{I_{0}} \cap \mathcal{X}_{0} \mid\left\{\left[\mathbb{X}^{\top} x_{T}^{\top}\right]^{\top} \in\left(\mathcal{S}_{\mathbb{}} \cap \mathcal{X}_{i m}^{T-1}\right) \times \mathcal{X}_{T} \mid\right.\right. \\
& \left.\left.E\left(I_{0}, x_{0}, \mathbb{}, \mathbb{X}, x_{T}\right)=0\right\} \neq \emptyset\right\}
\end{aligned}
$$


and further

$$
\mathcal{X}_{c}^{\rrbracket}(T, \mathcal{X}):=\bigcup_{I_{0} \in \mathcal{I}} \mathcal{X}_{c}^{I_{0} \rrbracket}(T, \mathcal{X}) .
$$

Thus, the set of $x_{0} \in \mathcal{X}_{0}$ for which there exists a $U \in \mathcal{R}^{m T}$ satisfying $\mathrm{C} 1$ ) under the initial state $x(0)=x_{0}$ (i.e., $\left.(I(0), x(0))=\left(I_{0}, x_{0}\right)\right)$ can be expressed as follows:

$$
\mathcal{X}_{c}(T, \mathcal{X}):=\bigcup_{\mathbb{1} \in \mathcal{I}^{T-1}} \mathcal{X}_{c}^{\llbracket}(T, \mathcal{X})
$$

where we note that $\mathcal{X}_{c}(T, \mathcal{X}) \subseteq \mathcal{X}_{0}$ because $\mathcal{X}_{c}^{I_{0} \rrbracket}(T, \mathcal{X})$ is defined as a subset of $\mathcal{X}_{0}$. Based on the aforementioned notation, we straightforwardly obtain a necessary and sufficient condition for $\Sigma$ to be $(T, \mathcal{X})$-controllable as follows.

Theorem 1: For the system $\Sigma$, suppose that $T \in\{2,3, \ldots\}$ and $\mathcal{X} \subseteq \mathcal{R}^{n(T+1)}$ are given. Then, $\Sigma$ is $(T, \mathcal{X})$-controllable if and only if the relation $\mathcal{X}_{c}(T, \mathcal{X})=\mathcal{X}_{0}$ holds.

It is remarked here that although a controllability condition based on a mixed-integer linear programming problem has been obtained in [5] and [6], Theorem 1 is derived from a slightly different point of view, i.e., it is obtained by directly characterizing a geometrical condition on the initial state set.

Next, based on Theorem 1, let us discuss the geometrical structure of the $(T, \mathcal{X})$-controllability space and how to check the $(T, \mathcal{X})$-controllability. First, as a straightforward result from [3] and [34], the following lemma is shown.

Lemma 2: For the system $\Sigma$, suppose that $T \in\{2,3, \ldots\}$ and $\mathcal{X} \subseteq \mathcal{R}^{n(T+1)}$ are given, and assume the following:

A1) $\mathcal{S}_{I} \cap \mathcal{X}_{k}$ is a polyhedron for every $I \in \mathcal{I}$ and $k \in\{0,1, \ldots, T-1\}$, and $\mathcal{X}_{T}$ is a polyhedron.

Then, $\mathcal{X}_{c}^{I_{0} \rrbracket}(T, \mathcal{X})$ is a polyhedron for every $\left(I_{0}, \llbracket\right) \in \mathcal{I}^{T}$.

Proof: This follows from the fact that $\mathcal{X}_{c}^{I_{0}}(T, \mathcal{X})$ is obtained by the projection of the set

$$
\begin{aligned}
& \mathcal{T}_{c}^{I_{0} \rrbracket}(T, \mathcal{X}):=\left\{\left[x_{0}^{\top} \mathbb{X}^{\top} x_{T}^{\top}\right]^{\top} \in\left(\mathcal{S}_{I_{0}} \cap \mathcal{X}_{0}\right) \times\left(\mathcal{S}_{\mathbb{}} \cap \mathcal{X}_{i m}^{T-1}\right)\right. \\
& \left.\times \mathcal{X}_{T} \mid E\left(I_{0}, x_{0}, \mathbb{}, \mathbb{X}, x_{T}\right)=0\right\}
\end{aligned}
$$

onto the $x_{0}$-space.

Lemma 2 implies that $\mathcal{X}_{c}^{I_{0} \rrbracket}(T, \mathcal{X})$ can be characterized as a polyhedron if A1) holds, from which as for the geometrical structure of $\mathcal{X}_{c}(T, \mathcal{X})$, it follows that $\mathcal{X}_{c}(T, \mathcal{X})$ is given as the union of $M^{T}$ polyhedra $\mathcal{X}_{c}^{I_{0}}(T, \mathcal{X})$. Therefore, although $\mathcal{X}_{c}(T, \mathcal{X})$ involves a complex structure, the condition $\mathcal{X}_{c}(T, \mathcal{X})=\mathcal{X}_{0}$ in Theorem 1 can be checked by computing $\mathcal{X}_{c}^{I_{0}}(T, \mathcal{X})$ based on the projection of $\mathcal{T}_{c}^{I_{0} \rrbracket}(T, \mathcal{X})$ in (9) and then by computing the union set defined as (7) and (8).

However, such a deterministic approach will not be practical from the viewpoint of computational complexity. More precisely, the following hold:

P1) as $T$ is taken larger, the required computation amount and memory in the computer become exponentially large because $M^{T}$ (too many!) polyhedra $\mathcal{X}_{c}^{I_{0}}(T, \mathcal{X})$ have to be explicitly characterized in the worst case.
In addition, the computation of each polyhedron $\mathcal{X}_{c}^{I_{0} \rrbracket}(T, \mathcal{X})$ is not so easy as follows:

P2) for the existing well-known projection algorithms such as Vertex enumeration, Fourier-Motzkin elimination (based on inequalities), and Block elimination (based on extreme rays) (see, e.g., [35], [36], and [10]), the computation amount for obtaining $\mathcal{X}_{c}^{I_{0}}(T, \mathcal{X})$ grows exponentially with $T$ and $n$ in general.

since their worst case computation amounts may exponentially grow with $n(T+1)$, i.e., the dimension of the space in which $\mathcal{T}_{c}^{I_{0}}(T, \mathcal{X})$ is defined. Moreover, the projection algorithms listed in P2) can deal only with open or closed polyhedra, although $\mathcal{X}_{c}^{I_{0} \rrbracket}(T, \mathcal{X})$ is, in general, neither open nor closed.

Example 1: Consider the system $\Sigma$ with $n:=2$ and $M:=$ 5 , where $A_{I}, B_{I}$, and $a_{I}$ are given as $A_{I}:=e^{A_{I}^{c} h}, B_{I}:=$ $\int_{0}^{h} e^{A_{I}^{c} t} d t B_{I}^{c}$, and $a_{I}:=\int_{0}^{h} e^{A_{I}^{c} t} d t a_{I}^{c}$ for the sampling period $h:=1$ and the continuous-time system parameters

$$
\begin{aligned}
A_{0}^{c} & :=\left[\begin{array}{cc}
-1 & 1 \\
0 & -1
\end{array}\right] \quad B_{0}^{c}:=\left[\begin{array}{cc}
-1 & 0 \\
0 & 0
\end{array}\right] \\
a_{0}^{c} & :=\left[\begin{array}{c}
-3 \\
5
\end{array}\right] \\
A_{1}^{c} & :=\left[\begin{array}{cc}
2 & -1 \\
1 & 2
\end{array}\right] \quad B_{1}^{c}:=\left[\begin{array}{cc}
1 & 1 \\
-1 & 0
\end{array}\right] \\
a_{1}^{c} & :=\left[\begin{array}{c}
0 \\
0
\end{array}\right] \\
A_{2}^{c} & :=\left[\begin{array}{cc}
-3 & 2 \\
0 & 0.5
\end{array}\right] \quad B_{2}^{c}:=\left[\begin{array}{ll}
1 & 0 \\
0 & 0
\end{array}\right] \\
a_{2}^{c} & :=\left[\begin{array}{cc}
1 \\
0
\end{array}\right] \\
A_{3}^{c} & :=\left[\begin{array}{cc}
0.5 & 0 \\
1 & -3
\end{array}\right] \quad B_{3}^{c}:=\left[\begin{array}{ll}
0 & 0 \\
1 & 0
\end{array}\right] \\
a_{3}^{c} & :=\left[\begin{array}{c}
-7 \zeta \\
0
\end{array}\right] \\
A_{4}^{c} & :=\left[\begin{array}{cc}
-0.1 & 0 \\
0 & -9
\end{array}\right] \quad B_{4}^{c}:=\left[\begin{array}{ll}
0 & 0 \\
0 & 0
\end{array}\right] \\
a_{4}^{c} & :=\left[\begin{array}{c}
2 \\
-5
\end{array}\right]
\end{aligned}
$$

where $\zeta \in\{1,2\}$, and $\mathcal{S}_{I}(I \in \mathcal{I})$ are given by

$$
\begin{aligned}
& \mathcal{S}_{0}:=\left\{x \in \mathcal{R}^{2} \mid\left[\begin{array}{cc}
1 & 0 \\
-1 & 0 \\
0 & 1 \\
0 & -1
\end{array}\right] x+\left[\begin{array}{l}
-10 \\
-10 \\
-10 \\
-10
\end{array}\right] \leq 0\right\} \\
& \mathcal{S}_{1}:=\left\{x \in \mathcal{R}^{2} \mid\left[\begin{array}{ll}
1 & 0
\end{array}\right] x+10<0\right\}
\end{aligned}
$$

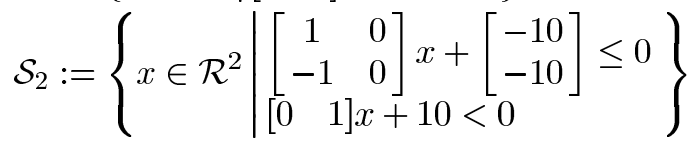

$$
\begin{aligned}
& \mathcal{S}_{3}:=\left\{x \in \mathcal{R}^{2} \mid \begin{array}{ll}
{\left[\begin{array}{ll}
0 & 1
\end{array}\right] x-10 \leq 0} \\
{\left[\begin{array}{ll}
-1 & 0
\end{array}\right] x+10<0}
\end{array}\right\} \\
& \mathcal{S}_{4}:=\left\{\begin{array}{l|l}
x \in \mathcal{R}^{2} & \begin{array}{ll}
{\left[\begin{array}{cc}
-1 & 0
\end{array}\right] x-10 \leq 0} \\
{\left[\begin{array}{cc}
0 & -1
\end{array}\right] x+10<0}
\end{array}
\end{array}\right\}
\end{aligned}
$$

as shown in Fig. 1. Note that the subsystem in only mode 1 is controllable in the usual sense. Then, for sev- 


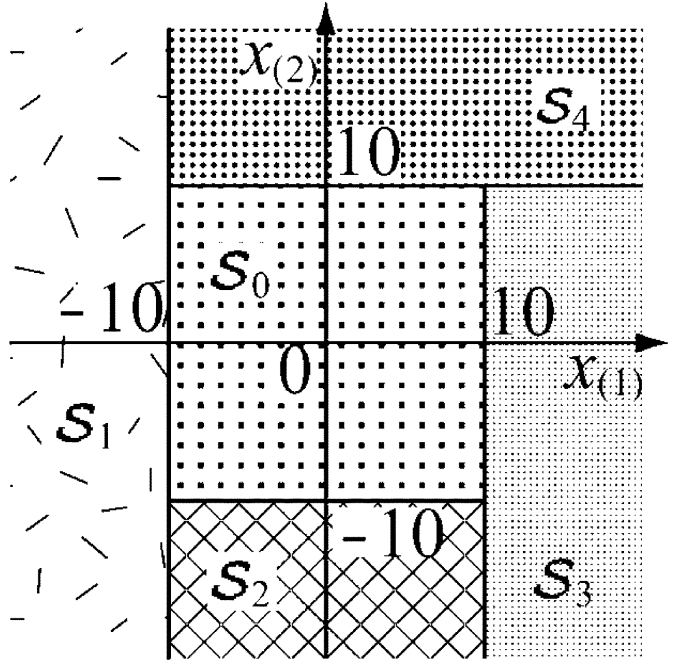

Fig. 1. Subregions assigned to each value of the mode in Example 1.

TABLE I

RESUlTS OF DETERMINISTIC CONTROLLABILITY ANALYSIS. (a) $\operatorname{CASE} \zeta=1$. (b) CASE $\zeta=2$

\begin{tabular}{|c||c|c|c|c|}
\hline & (a) \\
\hline$T$ & 3 & 4 & 5 & 6 \\
\hline \hline$(T, \mathcal{X})$-controllability & $\mathrm{N}$ & $\mathrm{N}$ & $\mathrm{N}$ & $?$ \\
\hline Computation time $[\mathrm{sec}]$ & 7.0 & 107.7 & 2865.1 & - \\
\hline
\end{tabular}

(b)

\begin{tabular}{|c||c|c|c|c|}
\hline$T$ & 3 & 4 & 5 & 6 \\
\hline \hline$(T, \mathcal{X})$-controllability & $\mathrm{N}$ & $\mathrm{Y}$ & $\mathrm{Y}$ & $?$ \\
\hline Computation time [sec] & 9.8 & 138.0 & 3544.1 & - \\
\hline
\end{tabular}

eral $T \in\{3,4,5,6\}$ and $\mathcal{X}_{0}:=[-20,20]^{2}, \mathcal{X}_{k}:=$ $\left[-10^{6}, 10^{6}\right]^{2}(k=1,2, \ldots, T-1)$ and $\mathcal{X}_{T}:=\{0\}$, which satisfy A1) in Lemma 2 , we have checked $\mathcal{X}_{c}(T, \mathcal{X})=\mathcal{X}_{0}$ in Theorem 1. The numerical results in determining the $(T, \mathcal{X})$-controllability are given in Table $\mathrm{I}$, where we used MATLAB (with GBT Toolbox [35]) on the computer with the Intel Pentium 4 2.53-GHz processor and the 2048-MB memory. The function "projpol" in [35], which is based on Vertex enumeration, was used to obtain $\mathcal{X}_{c}^{I_{0} \rrbracket}$ from $\mathcal{T}_{c}^{I_{0} \rrbracket}(T, \mathcal{X})$. The symbols " $Y$ " and " $N$ " express that $\Sigma$ is $(T, \mathcal{X})$-controllable and that $\Sigma$ is $(T, \mathcal{X})$-uncontrollable, respectively, and the symbol "?" implies that no answer can be obtained within 24 h. Table I shows that only for $T \in\{3,4,5\}$ (for a sufficiently small $T)$, the $(T, \mathcal{X})$-controllability problem can be solved in a practically short time.

It is remarked that, in this example, the enumeration-based approach of all the mode sequences is used in order to show the worst case computation amount, while some techniques to improve the computation amount, e.g., [7], can be used.

The aforementioned example motivates us to develop, as an alternative way, a probabilistic method for checking the $(T, \mathcal{X})$-controllability in the following sections.

\section{Probabilistic Controllability ANALYsis}

In this section, we propose a framework of approximately solving the $(T, \mathcal{X})$-controllability problem with a probabilistic accuracy.
Even for the problem involving the high computational complexity, such an approach will be hopeful to obtain some solution with a low complexity. In addition, although the probabilistic approach does not necessarily give a deterministic (strict) solution, an approximate answer guaranteed with a probabilistic accuracy must be useful. For example, if it is verified with a high probabilistic accuracy that a system is $(T, \mathcal{X})$-uncontrollable, we may consider to prepare an additional control input with the safety thinking. On the other hand, if it is verified with a high probabilistic accuracy that a system to be studied is $(T, \mathcal{X})$-controllable for some control input, then this will be useful for obtaining some information in considering its control problem; for example, this approach can give some policy in considering a dosage strategy at the experimental level of biosystems, e.g., gene regulatory networks which can be often modeled as the discrete-time PWA systems (see, e.g., [22] and [37]).

This section derives the following three types of randomized algorithms with their own approximate answers guaranteed with a probabilistic accuracy: a positive one-sided error algorithm (algorithm $\mathrm{P}$ ), which can determine that $\Sigma$ is $(T, \mathcal{X})$-controllable or is $(T, \mathcal{X})$-uncontrollable with a probabilistic accuracy, a negative one-sided error algorithm (algorithm $\mathrm{N}$ ), which can determine that $\Sigma$ is $(T, \mathcal{X})$-controllable with a probabilistic accuracy or is $(T, \mathcal{X})$-uncontrollable, and a two-sided error algorithm (algorithm $\mathrm{T}$ ), which can determine the $(T, \mathcal{X})$-controllability with a probabilistic accuracy. Then, it is shown that these algorithms are practical in terms of computational complexity, i.e., they are polynomial-time algorithms with respect to several problem variables. It should be remarked here that in the case that the system is linear (i.e., $M=1$ ), the decision results of these algorithms are consistent with the result from the usual controllability analysis for the linear systems, namely, if the algorithms are applied to the controllability problem of a linear system, the controllability is correctly determined (often approximately determined with a probabilistic accuracy). In the following subsections, for simplicity of notation, we suppose that $T$ and $\mathcal{X}$ are given in advance; so the symbols $\mathcal{X}_{c}^{I_{0}}, \mathcal{X}_{c}^{\natural}$, $\mathcal{X}_{c}$, and $\mathcal{T}_{c}^{I_{0} \rrbracket}$ are often used instead of $\mathcal{X}_{c}^{I_{0} \rrbracket}(T, \mathcal{X}), \mathcal{X}_{c}^{\llbracket}(T, \mathcal{X})$, $\mathcal{X}_{c}(T, \mathcal{X})$, and $\mathcal{T}_{c}^{I_{0}}(T, \mathcal{X})$, respectively.

\section{A. Probabilistic Controllability Analysis With Positive One-Sided Error}

We consider here the positive one-sided error algorithm (algorithm $\mathrm{P})$, which can determine if $\Sigma$ is $(T, \mathcal{X})$-controllable or $\Sigma$ is $(T, \mathcal{X})$-uncontrollable with a probabilistic accuracy. One of the key ideas to this approach is to adopt the random sampling of the mode sequence $\mathbb{\square} \in \mathcal{I}^{T-1}$.

1) Basic Algorithm: First, for a given single $x_{0} \in \mathcal{X}_{0}$, we consider a probabilistic condition such that there exists no $U \in$ $\mathcal{R}^{m T}$ satisfying C1) under the initial state $x(0)=x_{0}$. For given $x_{0} \in \mathcal{X}_{0}$, let

$$
\mathcal{I}_{c}^{T-1}\left(x_{0}\right):=\left\{\llbracket \in \mathcal{I}^{T-1} \mid x_{0} \in \mathcal{X}_{c}^{\square}\right\}
$$


This set expresses the set of $\llbracket \in \mathcal{I}^{T-1}$ for which there exists a $U \in \mathcal{R}^{m T}$ satisfying $\left[\begin{array}{llll}I(1) & I(2) & \cdots & I(T-1)\end{array}\right]^{\top}=\mathbb{\rrbracket}$ as well as C1) under the initial state $x(0)=x_{0}$. Then, if $\llbracket$ is an independent identically distributed (i.i.d.) random vector from the uniform distribution on $\mathcal{I}^{T-1}$, the relation

$$
\operatorname{Prob}\left\{\llbracket \in \mathcal{I}_{c}^{T-1}\left(x_{0}\right)\right\}=\frac{\operatorname{card}\left(\mathcal{I}_{c}^{T-1}\left(x_{0}\right)\right)}{\operatorname{card}\left(\mathcal{I}^{T-1}\right)}
$$

holds; so thanks to introducing the uniform distribution on $\mathcal{I}^{T-1}$, it follows that there does not exist a $U \in \mathcal{R}^{m T}$ satisfying $\mathrm{C} 1$ ) under the initial state $x(0)=x_{0}$ if and only if the probabilistic condition

$$
\operatorname{Prob}\left\{\llbracket \in \mathcal{I}_{c}^{T-1}\left(x_{0}\right)\right\}=0
$$

holds. Then, the following result is straightforwardly obtained from the result in [31]-[33].

Lemma 3: For the system $\Sigma$, suppose that $T \in\{2,3, \ldots\}$, $\mathcal{X} \subseteq \mathcal{R}^{n(T+1)}$, and $x_{0} \in \mathcal{X}_{0}$ are given. For given $\varepsilon_{P} \in(0,1)$ and $\delta_{P} \in(0,1)$, let

$$
N_{P}:=\left\lceil\frac{\ln \frac{1}{\delta_{P}}}{\ln \frac{1}{1-\varepsilon_{P}}}\right\rceil .
$$

Then, if the following holds:

C2) $\rrbracket^{i} \notin \mathcal{I}_{c}^{T-1}\left(x_{0}\right)$ holds for all $N_{P}$ i.i.d. random vectors $\rrbracket^{i}$ $\left(i=1,2, \ldots, N_{P}\right)$ from the uniform distribution on $\mathcal{I}^{T-1}$

the relation

$$
\operatorname{Prob}\left\{\operatorname{Prob}\left\{\llbracket \in \mathcal{I}_{c}^{T-1}\left(x_{0}\right)\right\} \leq \varepsilon_{P}\right\} \geq 1-\delta_{P}
$$

holds.

Lemma 3 implies that for given $x_{0} \in \mathcal{X}_{0}$, if C2) holds, the relation $\operatorname{card}\left(\mathcal{I}_{c}^{T-1}\left(x_{0}\right)\right) / \operatorname{card}\left(\mathcal{I}^{T-1}\right) \leq \varepsilon_{P}$ holds with probability greater than or equal to $1-\delta_{P}$. Note that (13) corresponds to the relation $\left(1-\varepsilon_{P}\right)^{N_{P}} \leq \delta_{P}$ and thus Prob $\{\llbracket \in$ $\left.\mathcal{I}_{c}^{T-1}\left(x_{0}\right)\right\}>\varepsilon_{P}$ implies that the probability that $\mathrm{C} 2$ ) holds is less than $\delta_{P}$, which proves Lemma 3 with a cumulative distribution function (see [31] for further mathematical details). Thus, if $\varepsilon_{P}$ and $\delta_{P}$ are sufficiently small, it is guaranteed with sufficiently high probability (greater than or equal to $1-\delta_{P}$ ) that for almost all $\square \in \mathcal{I}^{T-1}$ [i.e., for all $\llbracket \in \mathcal{I}^{T-1}$ except for elements in a subset of $\mathcal{I}^{T-1}$ whose cardinality is less than or equal to $\varepsilon_{P}$ card $\left.\left(\mathcal{I}^{T-1}\right)\right]$, there does not exist a $U \in \mathcal{R}^{m T}$ satisfying $\left[\begin{array}{llll}I(1) & I(2) & \cdots & I(T-1)\end{array}\right]^{\top}=\rrbracket$ and $\left.C 1\right)$ under the initial state $x(0)=x_{0}$. Therefore, (14) can be considered as a kind of relaxed condition of (12); in fact, (14) for $\varepsilon_{P}:=0$ and $\delta_{P}:=0$ is equivalent to (12).

Based on the previous discussion (in particular, based on (14)), we consider the $(T, \mathcal{X})$-controllability, i.e., whether for each $x_{0} \in \mathcal{X}_{0}$, there exists a $U \in \mathcal{R}^{m T}$ satisfying C1) under the initial state $x(0)=x_{0}$.
In order to verify that $\mathrm{C} 2$ ) holds for every $x_{0} \in \mathcal{X}_{0}$ at the same time, let us define

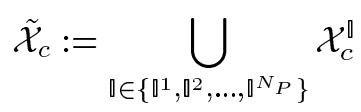

where $N_{P}$ is defined by (13) and $\rrbracket^{i}\left(i=1,2, \ldots, N_{P}\right)$ are i.i.d. random vectors from the uniform distribution on $\mathcal{I}^{T-1}$. Then, since $\tilde{\mathcal{X}}_{c} \subseteq \mathcal{X}_{c}$ holds, $\tilde{\mathcal{X}}_{c}=\mathcal{X}_{0}$ implies $\mathcal{X}_{c}=\mathcal{X}_{0}$ in Theorem 1 , i.e., $\Sigma$ is $(T, \mathcal{X})$-controllable. On the other hand, $\tilde{\mathcal{X}}_{c} \neq \mathcal{X}_{0}$ does not necessarily imply $\mathcal{X}_{c} \neq \mathcal{X}_{0}$, but can conclude that there exists an $x_{0} \in \mathcal{X}_{0}$ satisfying (14); such a system $\Sigma$ is called here to be $(\boldsymbol{T}, \mathcal{X})$-probabilistically-uncontrollable-with- $\left(\varepsilon_{P}, \delta_{P}\right)$ [or, simply, $(\boldsymbol{T}, \mathcal{X})$-p-uncontrollable-w- $\left.\left(\varepsilon_{\boldsymbol{P}}, \boldsymbol{\delta}_{\boldsymbol{P}}\right)\right]$. Therefore, by checking if $\tilde{\mathcal{X}}_{c}=\mathcal{X}_{0}$ holds or not, we can determine if $\Sigma$ is $(T, \mathcal{X})$-controllable or $(T, \mathcal{X})$-p-uncontrollable-w- $\left(\varepsilon_{P}, \delta_{P}\right)$.

The previous probabilistic controllability analysis is reasonable and practical in the following senses.

First, the $(T, \mathcal{X})$-p-uncontrollability-w- $\left(\varepsilon_{P}, \delta_{P}\right)$ for $\varepsilon_{P} \in(0,1)$ and $\delta_{P} \in(0,1)$ corresponds to a relaxed notion of the $(T, \mathcal{X})$-uncontrollability in the sense that it is consistent with the $(T, \mathcal{X})$-uncontrollability with the limit of $\varepsilon_{P}=\delta_{P}=0$. This follows from the two facts that $\Sigma$ is $(T, \mathcal{X})$-uncontrollable if and only if there exists an $x_{0} \in \mathcal{X}_{0}$ satisfying the probabilistic condition (12), and then (14) can be considered as a kind of relaxed condition of (12).

Second, even if it is obtained by the probabilistic analysis that $\Sigma$ is $(T, \mathcal{X})$-p-uncontrollable-w- $\left(\varepsilon_{P}, \delta_{P}\right)$, some useful policies for designing control systems are given. For example, we may consider that $\Sigma$ in question is faul intolerant, if it is decided to be $(T, \mathcal{X})$-p-uncontrollable-w- $\left(\varepsilon_{P}, \delta_{P}\right)$. In fact, in the case that $\Sigma$ cannot take some of the mode values due to breakdowns (such as mechanical switching faults), there does not necessarily exist a $U \in \mathcal{R}^{m T}$ satisfying C1) without going through these mode values, even when the normal system is $(T, \mathcal{X})$-controllable in the strict sense; so we can obtain a policy to determine if an additional control input applied to such a system is prepared with safety thinking. In addition, we can acquire the information on the hardness of checking the $(T, \mathcal{X})$-controllability from the probabilistic statement "there exists an $x_{0} \in \mathcal{X}_{0}$ satisfying (14)." The value $1-\operatorname{card}\left(\mathcal{I}_{c}^{T-1}\left(x_{0}\right)\right) / \operatorname{card}\left(\mathcal{I}^{T-1}\right)\left(=1-\operatorname{Prob}\left\{\emptyset \in \mathcal{I}_{c}^{T-1}\left(x_{0}\right)\right\}\right)$ corresponds to the worst case computation amount when we check by enumerating all the mode sequences, whether there exists a $U \in \mathcal{R}^{m T}$ satisfying C1) under $x(0)=x_{0}$ for a given single $x_{0} \in \mathcal{X}_{0}$ (e.g., if $1-\operatorname{card}\left(\mathcal{I}_{c}^{T-1}\left(x_{0}\right)\right) / \operatorname{card}\left(\mathcal{I}^{T-1}\right)=1$, i.e., $\operatorname{card}\left(\mathcal{I}_{c}^{T-1}\left(x_{0}\right)\right) / \operatorname{card}\left(\mathcal{I}^{T-1}\right)=0$, the condition $x_{0} \in \mathcal{X}_{c}^{I_{0}{ }^{C}}$ has to be verified for every $\rrbracket \in \mathcal{I}^{T-1}$ in the worst case, where $I_{0} \in \mathcal{I}$ satisfies $x_{0} \in \mathcal{S}_{I_{0}}$ ). Thus, the value $1-\inf _{x_{0} \in \mathcal{X}_{0}} \operatorname{Prob}\left\{\rrbracket \in \mathcal{I}_{c}^{T-1}\left(x_{0}\right)\right\}$ implies the worst case computation amount of the deterministic $(T, \mathcal{X})$-controllability analysis. Hence, the probabilistic statement in (14) implies that the worst case computation amount is greater than or equal to $1-\varepsilon_{P}$ with probability greater than or equal to $1-\delta_{P}$. This will give a policy for determining if the system itself is redesigned.

In this way, as an alternative way to the deterministic controllability analysis, the proposed probabilistic controllability analysis is useful in practical situations. 
Finally, the following randomized algorithm is shown for executing the aforementioned probabilistic controllability analysis.

\section{Algorithm P: Positive One-Sided Error Algorithm}

0: Given $T \in\{2,3, \ldots\}, \mathcal{X} \subseteq \mathcal{R}^{n(T+1)}, \varepsilon_{P} \in(0,1)$, and $\delta_{P} \in(0,1)$

1: Let $N_{P}$ be the number defined by (13);

2: Generate i.i.d. random vectors $\rrbracket^{1}, \rrbracket^{2}, \ldots, \rrbracket^{N_{P}}$ from the uniform distribution on $\mathcal{I}^{T-1}$;

3: $i:=1, \tilde{\mathcal{X}}_{c}(0):=\emptyset$;

4: $\mathcal{X}_{c}^{\square^{i}}:=\bigcup_{I_{0} \in \mathcal{I}} \mathcal{X}_{c}^{I_{0} \square^{i}}$

5: $\tilde{\mathcal{X}}_{c}(i):=\tilde{\mathcal{X}}_{c}(i-1) \cup \mathcal{X}_{c}^{n^{i}}$;

6: If $\tilde{\mathcal{X}}_{c}(i)==\mathcal{X}_{0}$ [i.e., there does not exist an $x_{0} \in \mathcal{X}_{0}$ satisfying $\mathrm{C} 2)]$ then Halt: return " $(T, \mathcal{X})$-controllable";

7: If $i==N_{P}$ then Halt: return

"( $(T, \mathcal{X})$-p-uncontrollable-w- $\left(\varepsilon_{P}, \delta_{P}\right)$ ";

8: $i:=i+1$, go to line 4 .

In algorithm $\mathrm{P}$, for given $\varepsilon_{P} \in(0,1)$ and $\delta_{P} \in(0,1)$, $N_{P}$ is defined by (13) (line 1 ), and $N_{P}$ i.i.d. random vectors $\square^{1}, \rrbracket^{2}, \ldots, \square^{N_{P}} \in \mathcal{I}^{T-1}$ are generated (line 2). Then, for each $\square^{i} \in \mathcal{I}^{T-1}\left(i=1,2, \ldots, N_{P}\right), \mathcal{X}_{c}^{\mathbb{i}^{i}}$ is computed according to (6) and (7) (line 4), and $\tilde{\mathcal{X}}_{c}(i)$ is defined as $\tilde{\mathcal{X}}_{c}(i-1) \cup \mathcal{X}_{c}^{n^{i}}$ (line 5). If $\tilde{\mathcal{X}}_{c}(i)=\mathcal{X}_{0}$ holds (this can be checked by standard polyhedral manipulation techniques, e.g., [10]) for some $i \in\left\{1,2, \ldots, N_{P}\right\}$, then $\mathcal{X}_{c}=\mathcal{X}_{0}$ holds; thus, the algorithm determines that $\Sigma$ is $(T, \mathcal{X})$-controllable (line 6). Otherwise, that is, $\tilde{\mathcal{X}}_{c}(i) \neq \mathcal{X}_{0}$ for all $i \in\left\{1,2, \ldots, N_{P}\right\}$, it determines that $\Sigma$ is $(T, \mathcal{X})$-p-uncontrollable-w- $\left(\varepsilon_{P}, \delta_{P}\right)$ (line 7). It is stressed that, in algorithm $\mathrm{P}$, a positive answer $(\Sigma$ is $(T, \mathcal{X})$-controllable $)$ is guaranteed in the strict sense.

Algorithm $\mathrm{P}$ terminates after computing at most $M N_{P}$ polyhedra $\mathcal{X}_{c}^{I_{0} \rrbracket}$, since $\mathcal{X}_{c}^{\rrbracket^{i}}$ is the union set of $M$ polyhedra $\mathcal{X}_{c}^{I_{0} \square^{i}}\left(I_{0} \in \mathcal{I}\right)$. Hence, algorithm $\mathrm{P}$ overcomes P1) in Section II-B. On the other hand, as shown in P2), each $\mathcal{X}_{c}^{I_{0}}$ d cannot be efficiently computed by the existing methods, i.e., their computation amounts exponentially grow with $T$ and $n$. Thus, a more efficient algorithm for computing $\mathcal{X}_{c}^{I_{0} \rrbracket}$ is required, which will be derived in Section III-A2.

Remark 1: In i.i.d. random vectors $\rrbracket^{i} \in \mathcal{I}^{T-1}(i=$ $\left.1,2, \ldots, N_{P}\right)$ used for algorithm $\mathrm{P}$, there may exist some infeasible $\llbracket^{i}$, i.e., for which there does not exist a $U \in \mathcal{R}^{m T}$ and an $x_{0} \in \mathcal{X}_{0}$ satisfying $\left[\begin{array}{llll}I(1) & I(2) & \cdots & I(T-1)\end{array}\right]^{\top}=\rrbracket^{i}$ under $x(0)=x_{0}$. Then, if the system is $(T, \mathcal{X})$-p-uncontrollable-w- $\left(\varepsilon_{P}, \delta_{P}\right)$, the value of $\varepsilon_{P}$ might correspond to the ratio of the feasible mode sequences, i.e., algorithm P sometimes gives the only information on the number of the feasible mode sequences; so infeasible $\square^{i}$ should not be used. On the other hand, since it must be hard to randomly sample only feasible $\rrbracket^{i}$ in a short time, $\square^{i} \in \mathcal{I}^{T-1}\left(i=1,2, \ldots, N_{P}\right)$ is used without distinction between feasible and infeasible in this paper. It is a limitation of the current version of the proposed method.

2) Subalgorithm for Computing $\mathcal{X}_{c}^{I_{0} \rrbracket}$ : Let us propose a polynomial-time algorithm with respect to $T$ for computing $\mathcal{X}_{c}^{I_{0}}$ under the following assumption:

\section{A2) $\mathcal{X}_{0}$ is bounded}

in addition to A1) in Lemma 2. This algorithm is derived according to the procedure that, at first, the closure of $\mathcal{X}_{c}^{I_{0} \rrbracket}$ (denoted by $\overline{\mathcal{X}}_{c}^{I_{0}}$ ) is obtained by projecting the closure of $\mathcal{T}_{c}^{I_{0} \rrbracket}$ in (9) onto the $x_{0}$-space, and next the set $\mathcal{X}_{c}^{I_{0}}$ is computed by eliminating unnecessary faces from $\overline{\mathcal{X}}_{c}^{I_{0}}$. In Section III-D (Lemma 12), it will be proven that it is a polynomial-time algorithm with respect to $T$.

First, the following results (Lemmas 4-7) are provided. Note that the proofs of all the results in this subsection are given in the Appendix.

Lemma 4: For the system $\Sigma$, suppose that $T \in\{2,3, \ldots\}$, $\mathcal{X} \subseteq \mathcal{R}^{n(T+1)}$, and $\left(I_{0}, 0\right) \in \mathcal{I}^{T}$ are given, and assume A1). Let $\epsilon_{1}$ denote the scalar variable and $C_{I_{0} \rrbracket}, \hat{C}_{I_{0} \rrbracket}$ and $D_{I_{0} \rrbracket}, \hat{D}_{I_{0} \rrbracket}$ denote matrices and vectors, respectively, satisfying the equation shown at the bottom of the page. Then, the following statements are equivalent:

1) $\mathcal{X}_{c}^{I_{0}} \neq \emptyset$;

2) the linear programming (LP) problem

- $\operatorname{LP} 1\left(I_{0}, \mathbb{0}\right)$

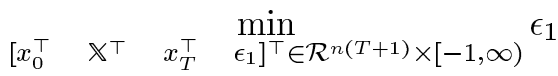

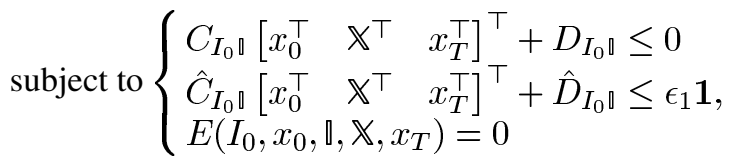

is feasible and its optimal value, denoted by $\epsilon_{1}^{*}$, is negative.

Lemma 4 implies that under A1), we can determine whether $\mathcal{X}_{c}^{I_{0} \rrbracket} \neq \emptyset$ or not by solving $\operatorname{LP} 1\left(I_{0}, \rrbracket\right)$. Note in $\operatorname{LP} 1\left(I_{0}, \rrbracket\right)$ that although the second constraint is not defined if the polyhedron $\left(\mathcal{S}_{I_{0}} \cap \mathcal{X}_{0}\right) \times\left(\mathcal{S}_{\mathbb{}} \cap \mathcal{X}_{i m}^{T-1}\right) \times \mathcal{X}_{T}$ is closed, we can determine if $\mathcal{X}_{c}^{I_{0} \rrbracket} \neq \emptyset$ by solving $\operatorname{LP} 1\left(I_{0}, \mathbb{\square}\right)$ in which the second constraint is ignored. Next, the following result is obtained.

Lemma 5: For the system $\Sigma$, suppose that $T \in\{2,3, \ldots\}$, $\mathcal{X} \subseteq \mathcal{R}^{n(T+1)},\left(I_{0}, \mathbb{0}\right) \in \mathcal{I}^{T}$, and a nonzero row vector $w \in$ $\mathcal{R}^{1 \times n}$ are given, and assume that A1), A2), and 1) in Lemma 4 hold. Let $\overline{\mathcal{T}}_{c}^{I_{0} \rrbracket}$ be the closure of $\mathcal{T}_{c}^{I_{0} \rrbracket}$ in (9), and let $x_{0}^{*}(w) \in \mathcal{R}^{n}$,

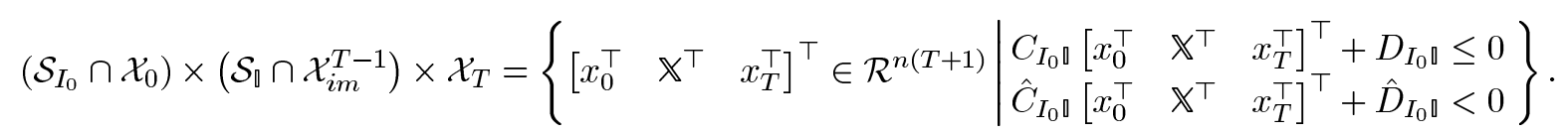


$\mathbb{X}^{*}(w) \in \mathcal{R}^{n(T-1)}$, and $x_{T}^{*}(w) \in \mathcal{R}^{n}$ be an optimal solution to the following LP problem:

- LP2 $(w)$

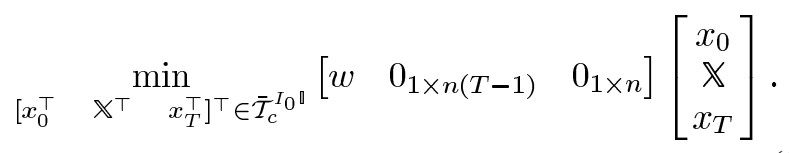

Then, $x_{0}^{*}(w)$ is a boundary point of $\overline{\mathcal{X}}_{c}^{I_{0} \rrbracket}$ (note that $\overline{\mathcal{X}}_{c}^{I_{0}}$ is the closure of $\mathcal{X}_{c}^{I_{0}}$ ).

Lemma 5 implies that if A1), A2), and 1) of Lemma 4 hold, some boundary points of $\overline{\mathcal{X}}_{c}^{I_{0}}$ are obtained from LP2 $(w)$ for arbitrarily given $w \in \mathcal{R}^{1 \times n}$. In fact, we can prove that the optimal value of LP2 $(w)$ is equal to that of the LP problem

$$
\min _{x_{0} \in \overline{\mathcal{X}}_{c}^{I_{0}}} w x_{0}
$$

and also that $x_{0}^{*}(w)$ is an optimal solution to the problem in (18) (see section B in the Appendix for the proof). Thus, a nonempty subset of $\overline{\mathcal{X}}_{c}^{I_{0}}$, denoted by $\tilde{\mathcal{X}}_{c}^{I_{0} \rrbracket}$, is provided as the convex hull of boundary points $x_{0}^{*}(w)$ of $\overline{\mathcal{X}}_{c}^{I_{0} \rrbracket}$ obtained by solving LP2 $2(w)$ for several $w$. Note here that the minimization problem in (18) cannot be directly solved because $\overline{\mathcal{X}}_{c}^{I_{0}}$ is not obtained in an explicit form at this point; on the other hand, $\overline{\mathcal{T}}_{c}^{I_{0}}$ in LP2 $2(w)$ is in advance given in an explicit form of (9). Furthermore, the following result is presented.

Lemma 6: For the system $\Sigma$, suppose that $T \in\{2,3, \ldots\}$, $\mathcal{X} \subseteq \mathcal{R}^{n(T+1)},\left(I_{0}, \mathbb{1}\right) \in \mathcal{I}^{T}$, and a nonempty, bounded, and closed polyhedron $\tilde{\mathcal{X}}_{c}^{I_{0} \rrbracket} \subseteq \overline{\mathcal{X}}_{c}^{I_{0} \rrbracket}$ are given, and assume that A1), A2), and 1) of Lemma 4 hold. Let $N_{w} \in \mathcal{N}_{+}, w_{i} \in \mathcal{R}^{1 \times n}$, and $v_{i} \in \mathcal{R}\left(i=1,2, \ldots, N_{w}\right)$ be a positive integer, row vectors, and scalars, respectively, satisfying

$$
\tilde{\mathcal{X}}_{c}^{I_{0} \rrbracket}=\left\{x_{0} \in \mathcal{R}^{n} \mid\left[\begin{array}{c}
w_{1} \\
w_{2} \\
\vdots \\
w_{N_{w}}
\end{array}\right] x_{0}+\left[\begin{array}{c}
v_{1} \\
v_{2} \\
\vdots \\
v_{N_{w}}
\end{array}\right] \leq 0\right\} .
$$

Then, the following statements hold.

1) The following statements are equivalent:

a) $\overline{\mathcal{X}}_{c}^{I_{0} \rrbracket}=\tilde{\mathcal{X}}_{c}^{I_{0} \rrbracket}$

b) the relation $w_{i} x_{0}^{*}\left(-w_{i}\right)+v_{i} \leq 0$ holds for every $i \in$ $\left\{1,2, \ldots, N_{w}\right\}$, where $x_{0}^{*}\left(-w_{i}\right)$ is defined in Lemma 5.

2) If the statement a) in 1) does not hold, the relation

$$
\begin{aligned}
\tilde{\mathcal{X}}_{c}^{I_{0} \rrbracket} & \subset \operatorname{hull}\left(\tilde{\mathcal{X}}_{c}^{I_{0} \rrbracket} \cup \bigcup_{w \in\left\{w_{1}, w_{2}, \ldots, w_{N_{w}}\right\}}\left\{x_{0}^{*}(-w)\right\}\right) \\
& \subseteq \overline{\mathcal{X}}_{c}^{I_{0} \rrbracket}
\end{aligned}
$$

holds.

Case 1) in Lemma 6 implies that under A1), A2), and 1) of Lemma 4 , we can determine if $\overline{\mathcal{X}}_{c}^{I_{0} \rrbracket}=\tilde{\mathcal{X}}_{c}^{I_{0}}$ holds for given $\tilde{\mathcal{X}}_{c}^{I_{0} \rrbracket}$, by solving $\operatorname{LP} 2\left(-w_{i}\right)$ for every $w_{i} \in \mathcal{R}^{1 \times n}\left(i=1,2, \ldots, N_{w}\right)$. On the other hand, 2) implies that if $\overline{\mathcal{X}}_{c}^{I_{0} \rrbracket} \neq \tilde{\mathcal{X}}_{c}^{I_{0} \rrbracket}, x_{0}^{*}\left(-w_{i}\right)$ for $i \in\left\{1,2, \ldots, N_{w}\right\}$ satisfying $w_{i} x_{0}^{*}\left(-w_{i}\right)+v_{i}>0$ is included in $\overline{\mathcal{X}}_{c}^{I_{0} \rrbracket}$ (from
Lemma 5), but not in $\tilde{\mathcal{X}}_{c}^{I_{0} \rrbracket}$, thus, we can obtain a new subset of $\overline{\mathcal{X}}_{c}^{I_{0} \rrbracket}$ strictly including $\tilde{\mathcal{X}}_{c}^{I_{0} \rrbracket}$.

Finally, we provide the following result.

Lemma 7: For the system $\Sigma$, suppose that $T \in\{2,3, \ldots\}$, $\mathcal{X} \subseteq \mathcal{R}^{n(T+1)}$, and $\left(I_{0}, \square\right) \in \mathcal{I}^{T}$ are given, and assume that $\left.\mathrm{A} 1\right)$, A2), and 1) of Lemma 4 hold. Let $N_{F} \in \mathcal{N}_{+}$be the number of the nonempty faces of $\overline{\mathcal{X}}_{c}^{I_{0} \rrbracket}, \mathcal{F}_{j}\left(\overline{\mathcal{X}}_{c}^{I_{0}}\right)\left(j=1,2, \ldots, N_{F}\right)$ (or, simply, $\mathcal{F}_{j}$ ) be a nonempty face of $\overline{\mathcal{X}}_{c}^{I_{0}}$, and $\mathcal{J}\left(\overline{\mathcal{X}}_{c}^{I_{0}}\right):=\{j \in$ $\left.\left\{1,2, \ldots, N_{F}\right\} \mid \mathcal{X}_{c}^{I_{0} \rrbracket} \cap \mathcal{F}_{j}\left(\overline{\mathcal{X}}_{c}^{I_{0} \rrbracket}\right)=\emptyset\right\}$. Then, the relation

$$
\mathcal{X}_{c}^{I_{0} \rrbracket}=\overline{\mathcal{X}}_{c}^{I_{0} \rrbracket}-\bigcup_{j \in \mathcal{J}\left(\overline{\mathcal{X}}_{c}^{I_{0} \rrbracket}\right)} \mathcal{F}_{j}\left(\overline{\mathcal{X}}_{c}^{I_{0} \rrbracket}\right)
$$

holds.

Lemma 7 implies that under A1), A2), and 1) of Lemma 4, the set $\mathcal{X}_{c}^{I_{0}}$ can be obtained from $\overline{\mathcal{X}}_{c}^{I_{0} \rrbracket}$. In fact, the right-hand side of (21) is computed as follows.

The faces $\mathcal{F}_{j}\left(\overline{\mathcal{X}}_{c}^{I_{0} \rrbracket}\right)\left(j=1,2, \ldots, N_{F}\right)$ can be obtained by applying standard polyhedral manipulation techniques to $\overline{\mathcal{X}}_{c}^{I_{0}}$. Next, $\mathcal{J}\left(\overline{\mathcal{X}}_{c}^{I_{0}}\right)$ can be also provided as follows. From the fact that one and only one of $\operatorname{ri}\left(\mathcal{F}_{j}\right) \subseteq \mathcal{X}_{c}^{I_{0} \rrbracket}$ or $\mathcal{X}_{c}^{I_{0} \rrbracket} \cap \operatorname{ri}\left(\mathcal{F}_{j}\right)=$ $\emptyset$ holds for each $j \in\left\{1,2, \ldots, N_{F}\right\}$ (see section D in the Appendix for the proof), it follows that $\mathcal{X}_{c}^{I_{0} \rrbracket} \cap \operatorname{ri}\left(\mathcal{F}_{j}\right)=\emptyset$ if and only if there exists an $x_{0} \in \operatorname{ri}\left(\mathcal{F}_{j}\right)$ satisfying $x_{0} \notin \mathcal{X}_{c}^{I_{0}}$. Furthermore, it follows from (6) that for given $x_{0} \in \mathcal{X}_{0}, x_{0} \in \mathcal{X}_{c}^{I_{0}}$ व holds if and only if the following LP problem:

- $\operatorname{LP} 3\left(I_{0}, x_{0}, \mathbb{\square}\right)$

$$
\begin{aligned}
& {\left[\begin{array}{ll}
\mathbb{X}^{\top} & \left.x_{T}^{\top} \quad \min _{\epsilon_{3}}\right]^{\top} \in \mathcal{R}^{n T} \times[-1, \infty) \\
\text { subject to } & \left\{\begin{array}{ll}
C_{\mathbb{0}}\left[\mathbb{X}^{\top}\right. & x_{T}^{\top}
\end{array}\right]^{\top}+D_{\mathbb{}} \leq 0 \\
\hat{C}_{\mathbb{0}}\left[\mathbb{X}^{\top}\right. & x_{T}^{\top} \\
E & ]^{\top}+\hat{D}_{\mathbb{}} \leq \epsilon_{3} \mathbf{1} \\
E\left(I_{0}, x_{0}, \mathbb{0}, \mathbb{X}, x_{T}\right)=0
\end{array}\right.}
\end{aligned}
$$

is feasible and its optimal value, denoted by $\epsilon_{3}^{*}$, is negative, where $\epsilon_{3}$ is a scalar variable and $C_{\mathbb{1}}, \hat{C}_{\mathbb{1}}$ and $D_{\mathbb{1}}, \hat{D}_{\mathbb{1}}$ are matrices and vectors, respectively, satisfying

$$
\begin{aligned}
& \left(\mathcal{S}_{\Downarrow} \cap \mathcal{X}_{i m}^{T-1}\right) \times \mathcal{X}_{T} \\
& =\left\{\begin{array}{l|l}
{\left[\mathbb{X}^{\top} x_{T}^{\top}\right]^{\top} \in \mathcal{R}^{n T} \mid \begin{array}{ll}
C_{\mathbb{0}}\left[\mathbb{X}^{\top}\right. & \left.x_{T}^{\top}\right]^{\top}+D_{\mathbb{1}} \leq 0 \\
\hat{C}_{\mathbb{1}}\left[\mathbb{X}^{\top}\right. & \left.x_{T}^{\top}\right]^{\top}+\hat{D}_{\mathbb{1}}<0
\end{array}}
\end{array}\right\} .
\end{aligned}
$$

These two facts imply that if there exists an $x_{0} \in \operatorname{ri}\left(\mathcal{F}_{j}\right)$ such that $\operatorname{LP} 3\left(I_{0}, x_{0}, \mathbb{\square}\right)$ is infeasible or $\epsilon_{3}^{*} \geq 0$, then $\mathcal{X}_{c}^{I_{0}} \cap$ $\operatorname{ri}\left(\mathcal{F}_{j}\right)=\emptyset$ holds; otherwise, $\mathcal{X}_{c}^{I_{0} \rrbracket} \cap \operatorname{ri}\left(\overline{\mathcal{F}}_{j}\right) \neq \emptyset$. Hence, by solving $\operatorname{LP} 3\left(I_{0}, x_{0}, \mathbb{}\right)$ for arbitrarily given $x_{0} \in \operatorname{ri}\left(\mathcal{F}_{j}\right)$, we can determine if $\mathcal{X}_{c}^{I_{0} \rrbracket} \cap \operatorname{ri}\left(\mathcal{F}_{j}\right)=\emptyset$ holds or not; thus, $\mathcal{J}\left(\overline{\mathcal{X}}_{c}^{I_{0}}\right)$ can be obtained by solving $\operatorname{LP} 3\left(I_{0}, x_{0}, \mathbb{\rrbracket}\right)$ with an $x_{0} \in \operatorname{ri}\left(\mathcal{F}_{j}\right)$ for every $\mathcal{F}_{j}\left(j=1,2, \ldots, N_{F}\right)$.

Based on the previous results, the proposed algorithm is given as follows.

\section{Subalgorithm 1: Computation Algorithm of $\mathcal{X}_{c}^{I_{0}}$}

0: Given $T \in\{2,3, \ldots\}, \mathcal{X} \subseteq \mathcal{R}^{n(T+1)}$, and $\left(I_{0}, \mathbb{}\right) \in \mathcal{I}^{T}$;

1: If $\mathcal{X}_{c}^{I_{0} \rrbracket}==\emptyset$ then $\mathcal{X}_{c}^{I_{0} \rrbracket}:=\emptyset$, go to line 4;

2: Derive $\overline{\mathcal{X}}_{c}^{I_{0} \rrbracket}$ (the closure of $\mathcal{X}_{c}^{I_{0} \rrbracket}$ ); 
2.1: Let $\tilde{\mathcal{X}}_{c}^{I_{0} \rrbracket}(0)$ be the convex hull of arbitrarily given boundary points of $\overline{\mathcal{X}}_{c}^{I_{0} \rrbracket}$

2.2: $i:=0$

2.3: Obtain $N_{w} \in \mathcal{N}_{+}, w_{1}, w_{2}, \ldots, w_{N_{w}} \in \mathcal{R}^{1 \times n}$, and $v_{1}, v_{2}, \ldots, v_{N_{w}} \in \mathcal{R}$ in (19) for $\tilde{\mathcal{X}}_{c}^{I_{0} \rrbracket}:=\tilde{\mathcal{X}}_{c}^{I_{0} \rrbracket}(i)$; (i.e., the

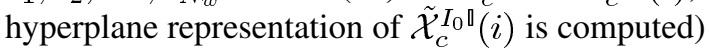

2.4: If $\overline{\mathcal{X}}_{c}^{I_{0} \rrbracket}==\tilde{\mathcal{X}}_{c}^{I_{0} \rrbracket}(i)$, then $\overline{\mathcal{X}}_{c}^{I_{0} \rrbracket}:=\tilde{\mathcal{X}}_{c}^{I_{0} \rrbracket}(i)$, go to line 3 ; else $\tilde{\mathcal{X}}_{c}^{I_{0} \rrbracket}(i+1):=\operatorname{hull}\left(\tilde{\mathcal{X}}_{c}^{I_{0} \rrbracket}(i) \cup\right.$ $\left.\bigcup_{w \in\left\{w_{1}, w_{2}, \ldots, w_{N_{w}}\right\}}\left\{x_{0}^{*}(-w)\right\}\right)$;

2.5: $i:=i+1$, go to line 2.3;

3: $\mathcal{X}_{c}^{I_{0} \rrbracket}:=\overline{\mathcal{X}}_{c}^{I_{0} \rrbracket}-\bigcup_{j \in \mathcal{J}\left(\overline{\mathcal{X}}_{c}^{I_{0}}\right)} \mathcal{F}_{j}\left(\overline{\mathcal{X}}_{c}^{I_{0} \rrbracket}\right) ;$

4: Halt: return $\mathcal{X}_{c}^{I_{0} \rrbracket}$.

In line 1 , the condition $\mathcal{X}_{c}^{I_{0} \rrbracket} \neq \emptyset$ is verified by Lemma 4 , i.e., it completes by solving $\operatorname{LP} 1\left(I_{0}, \mathbb{\square}\right)$. In line 2 , based on Lemmas 5 and 6 , the closure $\overline{\mathcal{X}}_{c}^{I_{0}}$ is obtained by generating a sequence of sets $\tilde{\mathcal{X}}_{c}^{I_{0} \rrbracket}(0), \tilde{\mathcal{X}}_{c}^{I_{0} \rrbracket}(1), \ldots, \tilde{\mathcal{X}}_{c}^{I_{0} \rrbracket}\left(i_{f}\right)$ satisfying the relation of the strict inclusion

$$
\tilde{\mathcal{X}}_{c}^{I_{0} \rrbracket}(0) \subset \tilde{\mathcal{X}}_{c}^{I_{0} \rrbracket}(1) \subset \cdots \subset \tilde{\mathcal{X}}_{c}^{I_{0} \rrbracket}\left(i_{f}\right)=\overline{\mathcal{X}}_{c}^{I_{0} \rrbracket}
$$

for a finite number $i_{f} \in \mathcal{N}$. From Lemma $5, \tilde{\mathcal{X}}_{c}^{I_{0}}(0)$ in line 2.1 can be obtained by solving LP2 $(w)$ for arbitrarily given $w \in$ $\mathcal{R}^{1 \times n}$. Then, by Lemma $6, \overline{\mathcal{X}}_{c}^{I_{0}}$ is derived by repeating the procedure of lines 2.3-2.5 for $i=0,1, \ldots, i_{f}$ : If $\overline{\mathcal{X}}_{c_{0}}^{I_{0}}=\tilde{\mathcal{X}}_{c}^{I_{0} \rrbracket}(i)$ [i.e., b) in 1) of Lemma 6 holds for $\left.\tilde{\mathcal{X}}_{c}^{I_{0} \rrbracket}:=\tilde{\mathcal{X}}_{c}^{I_{0}}(i)\right]$, then $\overline{\mathcal{X}}_{c}^{I_{0} \rrbracket}$ can be obtained as $\tilde{\mathcal{X}}_{c}^{I_{0} \rrbracket}(i)$, otherwise, $\tilde{\mathcal{X}}_{c}^{I_{0} \rrbracket}(i+1)$ is defined as $\operatorname{hull}\left(\tilde{\mathcal{X}}_{c}^{I_{0} \rrbracket}(i) \cup \bigcup_{w \in\left\{w_{1}, w_{2}, \ldots, w_{N_{w}}\right\}}\left\{x_{0}^{*}(-w)\right\}\right)$, where $w_{1}, w_{2}, \ldots, w_{N_{w}}$ are given by (19) for $\mathcal{X}_{c}^{I_{0} \rrbracket}:=\tilde{\mathcal{X}}_{c}^{I_{0} \rrbracket}(i)$. Note that by virtue of the relation (20), (23) holds. Finally, in line 3 , the set $\mathcal{X}_{c}^{I_{0}}$ is derived from $\overline{\mathcal{X}}_{c}^{I_{0} \rrbracket}$ by using Lemma 7 . In the following example, we show how $\mathcal{X}_{c}^{I_{0} 0}$ is obtained by subalgorithm 1.

Example 2: Consider the polyhedron shown in (24) at the bottom of the page, whose projection onto the $x_{0}$-space is equal to the polyhedron

$$
\mathcal{X}_{c}^{I_{0} \rrbracket}=\left\{\begin{array}{l|l}
x_{0} \in \mathcal{R}^{2} & \begin{array}{l}
{\left[\begin{array}{c}
\tilde{w}_{1} \\
\tilde{w}_{2} \\
\tilde{w}_{3}
\end{array}\right] x_{0}+\left[\begin{array}{c}
\tilde{v}_{1} \\
\tilde{v}_{2} \\
\tilde{v}_{3}
\end{array}\right] \leq 0} \\
{\left[\begin{array}{c}
\tilde{w}_{4} \\
\tilde{w}_{5}
\end{array}\right] x_{0}+\left[\begin{array}{l}
\tilde{v}_{4} \\
\tilde{v}_{5}
\end{array}\right]<0}
\end{array}
\end{array}\right\}
$$

as shown in Fig. 2(a), where $\hat{w}_{i j} \in \mathcal{R}^{1 \times 2}, \hat{v}_{i} \in \mathcal{R}(i=$ $1,2, \ldots, 8, j=1,2,3)$ and $\tilde{w}_{l} \in \mathcal{R}^{1 \times 2}, \tilde{v}_{l} \in \mathcal{R}(l=$ $1,2, \ldots, 5)$. We discuss here how $\mathcal{X}_{c}^{I_{0} \rrbracket}$ in (25) is obtained from (24).

In line 1 , we conclude $\mathcal{X}_{c}^{I_{0} \rrbracket} \neq \emptyset$ by solving $\operatorname{LP} 1\left(I_{0}, \llbracket\right)$ defined as

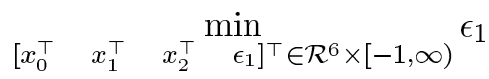

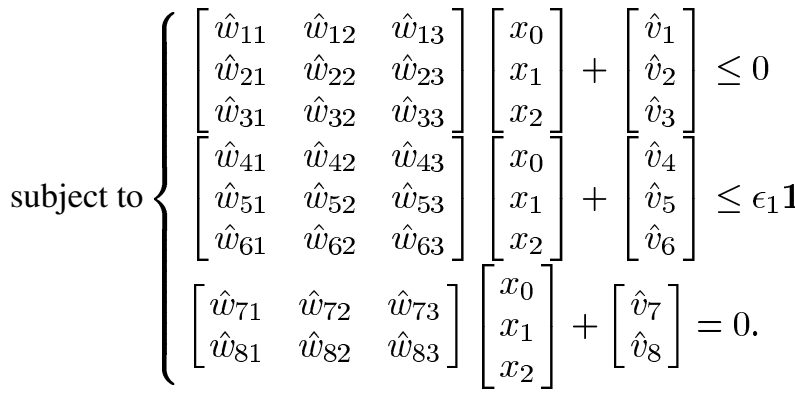

In line 2.1, suppose that $\tilde{\mathcal{X}}_{c}^{I_{0}}(0)$ is given as

$$
\begin{aligned}
\tilde{\mathcal{X}}_{c}^{I_{0}}(0):=\operatorname{hull}\left(\left\{x_{0}^{*}\left(\left[\begin{array}{ll}
0 & -1
\end{array}\right]\right), x_{0}^{*}\left(\left[\begin{array}{ll}
-1 & 0
\end{array}\right]\right),\right.\right. \\
\left.\left.x_{0}^{*}\left(\left[\begin{array}{ll}
0 & 1
\end{array}\right]\right), x_{0}^{*}\left(\left[\begin{array}{ll}
1 & 0
\end{array}\right]\right)\right\}\right)
\end{aligned}
$$

which is the convex hull of $x_{0}^{*}(w)$ obtained by $\operatorname{LP} 2(w)$ for $w=\left[\begin{array}{ll}0 & -1\end{array}\right],\left[\begin{array}{ll}-1 & 0\end{array}\right],\left[\begin{array}{ll}0 & 1\end{array}\right],\left[\begin{array}{ll}1 & 0\end{array}\right]$ (arbitrarily given). Then,

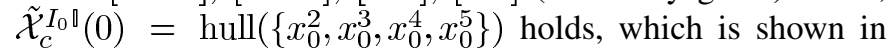
Fig. 2(b). After setting $i:=0$ in line 2.2, $N_{w}, w_{1}, w_{2}, \ldots, w_{N_{w}}$, and $v_{1}, v_{2}, \ldots, v_{N_{w}}$ are derived in line 2.3 as follows:

$$
\tilde{\mathcal{X}}_{c}^{I_{0} \rrbracket}(0)=\left\{x_{0} \in \mathcal{R}^{2} \mid\left[\begin{array}{c}
\tilde{w}_{2} \\
\tilde{w}_{3} \\
\tilde{w}_{4} \\
\tilde{w}_{\alpha}
\end{array}\right] x_{0}+\left[\begin{array}{c}
\tilde{v}_{2} \\
\tilde{v}_{3} \\
\tilde{v}_{4} \\
\tilde{v}_{\alpha}
\end{array}\right] \leq 0\right\}
$$

where the line of $\tilde{w}_{\alpha} x_{0}+\tilde{v}_{\alpha}=0\left(\tilde{w}_{\alpha} \in \mathcal{R}^{1 \times 2}, \tilde{v}_{\alpha} \in \mathcal{R}\right)$ is given by $(\alpha)$ in Fig. 2(b). In line 2.4, $\overline{\mathcal{X}}_{c}^{I_{0} \rrbracket} \neq \tilde{\mathcal{X}}_{c}^{I_{0}}(0)$ is shown by checking the condition b) in 1) of Lemma 6 for $\tilde{\mathcal{X}}_{c}^{I_{0} \rrbracket}:=$ $\tilde{\mathcal{X}}_{c}^{I_{0} \rrbracket}(0)$. In fact, we have $\tilde{w}_{l} x_{0}^{*}\left(-\tilde{w}_{l}\right)+\tilde{v}_{l} \leq 0(l=2,3,4)$

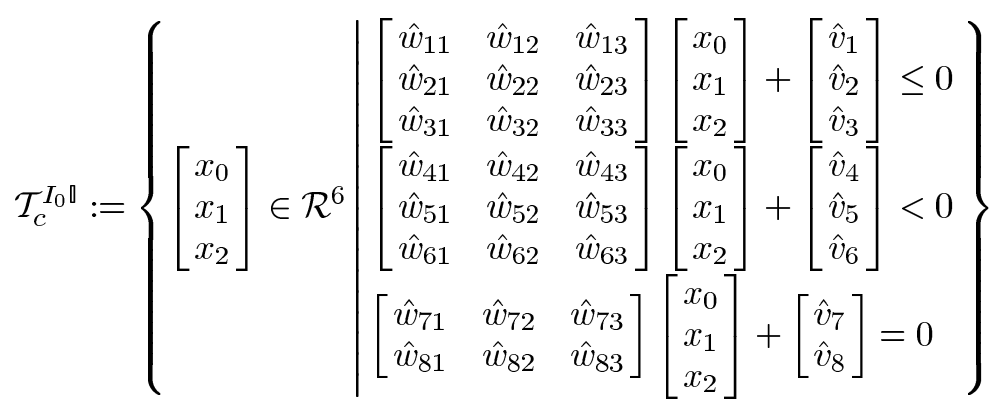




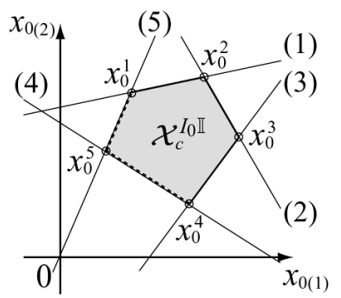

(1) $\quad \tilde{w}_{1} x_{0}+\tilde{v}_{1}=0$

(2) $\tilde{w}_{2} x_{0}+\tilde{v}_{2}=0$

(3) $\tilde{w}_{3} x_{0}+\tilde{v}_{3}=0$

(4) $\tilde{w}_{4} x_{0}+\tilde{v}_{4}=0$

(5) $\tilde{w}_{5} x_{0}+\tilde{v}_{5}=0$

(a)

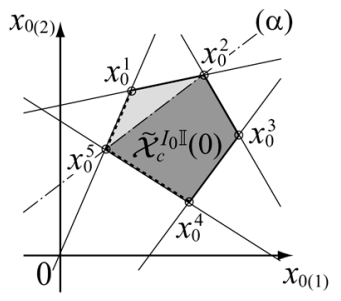

(a) $\quad \tilde{w}_{\alpha} x_{0}+\tilde{v}_{\alpha}=0$

(b)

Fig. 2. Sets $\mathcal{X}_{c}^{I_{0} \rrbracket}$ and $\tilde{\mathcal{X}}_{c}^{I_{0}}(0)$ in Example 2. (a) Set $\mathcal{X}_{c}^{I_{0}}$. (b) Set $\tilde{\mathcal{X}}_{c}^{I_{0}}(0)$.

and $\tilde{w}_{\alpha} x_{0}^{*}\left(-\tilde{w}_{\alpha}\right)+\tilde{v}_{\alpha}>0$ (note that $\left.x_{0}^{*}\left(-\tilde{w}_{\alpha}\right)=x_{0}^{1}\right)$. Thus,

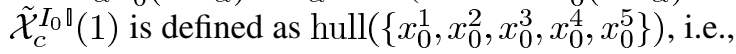

$$
\left.\tilde{\mathcal{X}}_{c}^{I_{0} \rrbracket}(1)=\left\{x_{0} \in \mathcal{R}^{2} \mid \begin{array}{c}
\tilde{w}_{1} \\
\tilde{w}_{2} \\
\tilde{w}_{3} \\
\tilde{w}_{4} \\
\tilde{w}_{5}
\end{array}\right] x_{0}+\left[\begin{array}{c}
\tilde{v}_{1} \\
\tilde{v}_{2} \\
\tilde{v}_{3} \\
\tilde{v}_{4} \\
\tilde{v}_{5}
\end{array}\right] \leq 0\right\} .
$$

In line 2.5 , the counter $i$ is incremented as $i:=1$ and the procedure goes to line 2.3. In lines 2.3 and 2.4 for $i=1$, after some calculation similar to the previous procedure, we conclude $\overline{\mathcal{X}}_{c}^{I_{0} \rrbracket}=\tilde{\mathcal{X}}_{c}^{I_{0}}(1)$ and it goes to line 3 .

In line 3,11 nonempty faces of $\overline{\mathcal{X}}_{c}^{I_{0} \rrbracket}$ (i.e., $N_{F}=11$ ) are given as $\mathcal{F}_{1}:=\left\{x_{0}^{1}\right\}, \mathcal{F}_{2}:=\left\{x_{0}^{2}\right\}, \ldots, \mathcal{F}_{5}:=\left\{x_{0}^{5}\right\}, \mathcal{F}_{6}:=\overline{\mathcal{X}}_{c}^{I_{0} \rrbracket} \cap$ $\left\{x_{0} \in \mathcal{R}^{2} \mid \tilde{w}_{1} x_{0}+\tilde{v}_{1}=0\right\}, \mathcal{F}_{7}:=\overline{\mathcal{X}}_{c}^{I_{0} \rrbracket} \cap\left\{x_{0} \in \mathcal{R}^{2} \mid \tilde{w}_{2} x_{0}+\right.$ $\left.\tilde{v}_{2}=0\right\}, \ldots, \mathcal{F}_{10}:=\overline{\mathcal{X}}_{c}^{I_{0} 0} \cap\left\{x_{0} \in \mathcal{R}^{2} \mid \tilde{w}_{5} x_{0}+\tilde{v}_{5}=0\right\}$, and $\mathcal{F}_{11}:=\overline{\mathcal{X}}_{c}^{I_{0}}$. Then, we have $\mathcal{J}\left(\overline{\mathcal{X}}_{c}^{I_{0}}\right)=\{1,4,5,9,10\}$ by solving $\operatorname{LP} 3\left(I_{0}, x_{0}, \mathbb{0}\right)$ in (22) with arbitrarily given $x_{0} \in$ $\operatorname{ri}\left(\mathcal{F}_{i}\right)$ for each $i \in\left\{1,2, \ldots, N_{F}\right\}$. Thus, by means of Lemma 7 , we conclude that $\mathcal{X}_{c}^{I_{0}}$ is given as (25). This completes the procedure for obtaining $\mathcal{X}_{c}^{I_{0} \rrbracket}$ in subalgorithm 1 .

Finally, we show when subalgorithm 1 terminates. As shown in (23), $i_{f} \in \mathcal{N}$ expresses the value of $i$ at the end of subalgorithm 1. Let $\mathcal{E}(i)$ be the set of $j \in\left\{1,2, \ldots, N_{F}\right\}$ satisfying $\tilde{\mathcal{X}}_{c}^{I_{0}}(i) \cap \mathcal{F}_{j} \neq \emptyset$ in subalgorithm 1. Then, since

$$
\operatorname{card}(\mathcal{E}(0))<\operatorname{card}(\mathcal{E}(1))<\cdots<\operatorname{card}\left(\mathcal{E}\left(i_{f}\right)\right)=N_{F}
$$

holds (see section $\mathrm{E}$ in the Appendix for the proof), we have

$$
i_{f} \leq N_{F} .
$$

Therefore, subalgorithm 1 terminates as follows.

Lemma 8: For the system $\Sigma$, suppose that $T \in\{2,3, \ldots\}$, $\mathcal{X} \subseteq \mathcal{R}^{n(T+1)},\left(I_{0}, \mathbb{}\right) \in \mathcal{I}^{T}$ are given, and A1) and A2) hold. Let $N_{0}$ be the number of arbitrarily given row vectors $w \in$ $\mathcal{R}^{1 \times n}$ for obtaining $\tilde{\mathcal{X}}_{c}^{I_{0}}(0)$ and let $N_{w}(i)\left(i=0,1, \ldots, i_{f}\right)$ be the value of $N_{w}$ for $\tilde{\mathcal{X}}_{c}^{I_{0} \rrbracket}:=\tilde{\mathcal{X}}_{c}^{I_{0} \rrbracket}(i)$. Then, subalgorithm 1 terminates after solving at most $1+N_{0}+\sum_{i=0}^{N_{F}} N_{w}(i)+N_{F}$ LP problems and $1+N_{F}$ convex hull problems (i.e., to derive the hyperplane representation of $\tilde{\mathcal{X}}_{c}^{I_{0}}(i)$ ), namely, it terminates in a finite time.

Note that each term of $1+N_{0}+\sum_{i=0}^{N_{F}} N_{w}(i)+N_{F}$ expresses the number of each LP problem solved in lines 1, 2.1, 2.4, and 3. Hereafter, algorithm P including subalgorithm 1 is simply called algorithm P.

Remark 2: The projection method similar to the previous algorithm (see [38] for the conference paper), which is called the iterative hull method, has been independently proposed in MPT toolbox [10] by Raković and Mayne. In addition, the algorithm called the equality set projection algorithm [39] has been recently proposed, while the complexity for degenerate polyhedra, however, has never been analyzed, and it has been described in [39] that other projection algorithms (e.g., Vertex enumeration, Fourier-Motzkin elimination, and Block elimination) may be more efficient for degenerate polyhedra. Note that $\overline{\mathcal{T}}_{c}^{I_{0}}$ is often degenerate.

Remark 3: Subalgorithm 1 is an efficient algorithm for projecting polyhedra that are not necessarily bounded, nondegenerate, and/or open/closed; so it is very useful for various types of projection problems including the problem addressed here. Of course, it can be applied to the deterministic controllability analysis discussed in Section II.

\section{B. Probabilistic Controllability Analysis With Negative One-Sided Error}

We consider the negative one-sided error algorithm (algorithm $\mathrm{N}$ ), which can determine if $\Sigma$ is $(T, \mathcal{X})$-controllable with a probabilistic accuracy or $\Sigma$ is $(T, \mathcal{X})$-uncontrollable. One of the key ideas here is to adopt the random sampling of the initial state $x_{0} \in \mathcal{X}_{0}$ under the following assumptions.

A3) The set $\mathcal{X}_{0}$ is bounded and measurable.

A4) The measure of $\mathcal{X}_{0}$ is not zero (i.e., $\operatorname{vol}\left(\mathcal{X}_{0}\right) \neq 0$ ).

Assumption A3) is necessary to define the measures of $\mathcal{X}_{0}$ and its subsets, which is an essential assumption for the approach proposed here. In contrast, (A4) is assumed for simplicity of discussion; thus, the following approach can be easily extended to the case that the measure of $\mathcal{X}_{0}$ is zero. In fact, for the case that $\mathcal{X}_{0}$ is a closed polyhedron of measure zero in $\mathcal{R}^{n}$, the following discussion straightforwardly holds in the $\operatorname{dim}\left(\mathcal{X}_{0}\right)$-dimensional space including $\mathcal{X}_{0}$ instead of $\mathcal{R}^{n}$.

First, as a preliminary, the following result is obtained.

Lemma 9: For the system $\Sigma$, suppose that $T \in\{2,3, \ldots\}$, $\mathcal{X} \subseteq \mathcal{R}^{n(T+1)}$, and $x_{0} \in \mathcal{X}_{0}$, which provides $I_{0} \in \mathcal{I}$ satisfying $x_{0} \in \mathcal{S}_{I_{0}}$, are given. Then, the following statements are equivalent:

1) $x_{0} \in \mathcal{X}_{c}$

2) there exists an $\square \in \mathcal{I}^{T-1}$ such that $\operatorname{LP} 3\left(I_{0}, x_{0}, \mathbb{\square}\right)$ in (22) is feasible and the optimal value $\epsilon_{3}^{*}$ is negative.

Proof: For given $x_{0} \in \mathcal{X}_{0}$ (which specifies $I_{0}$ ) and $\rrbracket \in$ $\mathcal{I}^{T-1}$, the relation $x_{0} \in \mathcal{X}_{c}^{I_{0} \rrbracket}$ holds if and only if $\operatorname{LP} 3\left(I_{0}, x_{0}, \rrbracket\right)$ is feasible and $\epsilon_{3}^{*}<0$. Thus, it follows from (7) and (8) that 1) is equivalent to 2 ). 
Lemma 9 implies that for given $x_{0} \in \mathcal{X}_{0}$, the condition $x_{0} \in \mathcal{X}_{c}$ can be checked by solving $\operatorname{LP} 3\left(I_{0}, x_{0}, \mathbb{\square}\right)$ for every $\llbracket \in \mathcal{I}^{T-1}$. Based on this fact, the following negative one-sided error algorithm is proposed. Let $x_{0}$ be a random vector with the uniform probability density function $\phi_{x_{0}}$ on $\mathcal{X}_{0}$; so under A3) and A4), we formally define

$$
\operatorname{Prob}\left\{x_{0} \in \mathcal{X}_{c}\right\}:=\int_{\mathcal{X}_{c}} \phi_{x_{0}} d x_{0} .
$$

Note that $\operatorname{Prob}\left\{x_{0} \in \mathcal{X}_{0}-\mathcal{X}_{c}\right\}=1-\operatorname{Prob}\left\{x_{0} \in \mathcal{X}_{c}\right\}$ holds; so the following result is obtained in a similar way to the case of Lemma 3.

Lemma 10: For the system $\Sigma$, suppose that $T \in\{2,3, \ldots\}$ and $\mathcal{X} \subseteq \mathcal{R}^{n(T+1)}$ are given, and assume A3) and A4). For given $\varepsilon_{N} \in(0,1)$ and $\delta_{N} \in(0,1)$, let

$$
N_{N}:=\left\lceil\frac{\ln \frac{1}{\delta_{N}}}{\ln \frac{1}{1-\varepsilon_{N}}}\right\rceil \text {. }
$$

Then, if the following holds:

C3) $x_{0}^{i} \in \mathcal{X}_{c}$ holds for all $N_{N}$ i.i.d. random vectors $x_{0}^{i}(i=$ $\left.1,2, \ldots, N_{N}\right)$ from the uniform distribution on $\mathcal{X}_{0}$

the relation

$$
\operatorname{Prob}\left\{\operatorname{Prob}\left\{x_{0} \in \mathcal{X}_{0}-\mathcal{X}_{c}\right\} \leq \varepsilon_{N}\right\} \geq 1-\delta_{N}
$$

holds.

Lemma 10 implies that if $\mathrm{C} 3)$ holds, $\operatorname{vol}\left(\mathcal{X}_{0}-\mathcal{X}_{c}\right) / \operatorname{vol}\left(\mathcal{X}_{0}\right) \leq$ $\varepsilon_{N}$ holds with probability greater than or equal to $1-\delta_{N}$, since the relation

$$
\operatorname{Prob}\left\{x_{0} \in \mathcal{X}_{c}\right\}=\frac{\operatorname{vol}\left(\mathcal{X}_{c}\right)}{\operatorname{vol}\left(\mathcal{X}_{0}\right)}
$$

holds. Thus, if $\varepsilon_{N}$ and $\delta_{N}$ are sufficiently small, it is guaranteed with sufficiently high probability (greater than or equal to $1-\delta_{N}$ ) that for almost all $x_{0} \in \mathcal{X}_{0}$ (i.e., for all $x_{0} \in \mathcal{X}_{0}$ except for elements in a subset of $\mathcal{X}_{0}$ whose volume is less than or equal to $\varepsilon_{N} \operatorname{vol}\left(\mathcal{X}_{0}\right)$ ), there exists a $U \in \mathcal{R}^{m T}$ satisfying $\mathrm{C} 1$ ) under the initial state $x(0)=x_{0}$. Since the condition $\operatorname{vol}\left(\mathcal{X}_{c}\right) / \operatorname{vol}\left(\mathcal{X}_{0}\right)=1$ corresponds to a relaxed version of the $(T, \mathcal{X})$-controllability condition in Theorem $1,(31)$ also corresponds to a relaxed condition of the condition in Theorem 1. Thus, $\Sigma$ satisfying (31) is said here to be $(\boldsymbol{T}, \mathcal{X})$-probabilistically-controllable-with- $\left(\varepsilon_{N}, \delta_{N}\right)$ (or simply, $(\boldsymbol{T}, \mathcal{X})$-p-controllable-w- $\left.\left(\varepsilon_{N}, \delta_{N}\right)\right)$. If C3) does not hold, on the other hand, then $\mathcal{X}_{c} \neq \mathcal{X}_{0}$ deterministically holds, namely, $\Sigma$ is $(T, \mathcal{X})$-uncontrollable. In this way, we can determine if $\Sigma$ is $(T, \mathcal{X})$-p-controllable-w- $\left(\varepsilon_{N}, \delta_{N}\right)$ or $(T, \mathcal{X})$-uncontrollable.

Based on the previous discussion, the following algorithm is proposed under A3) and A4).

\section{Algorithm N: Negative One-Sided Error Algorithm}

0: Given $T \in\{2,3, \ldots\}, \mathcal{X} \subseteq \mathcal{R}^{n(T+1)}, \varepsilon_{N} \in(0,1)$, and $\delta_{N} \in(0,1)$;
1: Let $N_{N}$ be the number defined by (30);

2: Generate i.i.d. random vectors $x_{0}^{1}, x_{0}^{2}, \ldots, x_{0}^{N_{N}}$ from the uniform distribution on $\mathcal{X}_{0}$;

3: $i:=1$;

4: If $x_{0}^{i} \notin \mathcal{X}_{c}$ [i.e., C3) does not hold], then Halt: return "( $T, \mathcal{X})$-uncontrollable";

5: If $i==N_{N}$ then Halt: return

" $(T, \mathcal{X})$-p-controllable-w- $\left(\varepsilon_{N}, \delta_{N}\right)$ ";

6: $i:=i+1$, go to line 4 .

In algorithm $\mathrm{N}$, for given $\varepsilon_{N} \in(0,1)$ and $\delta_{N} \in(0,1)$, $N_{N}$ is defined by (30) (line 1), and $N_{N}$ i.i.d. random vectors $x_{0}^{1}, x_{0}^{2}, \ldots, x_{0}^{N_{N}} \in \mathcal{X}_{0}$ are generated (line 2). Then, if $x_{0}^{i} \notin$ $\mathcal{X}_{c}$ holds [i.e., 2) of Lemma 9 does not hold] for some $i \in$ $\left\{1,2, \ldots, N_{N}\right\}$, the algorithm determines that $\Sigma$ is $(T, \mathcal{X})$-uncontrollable (line 4$)$. In this case, $\mathcal{X}_{c} \neq \mathcal{X}_{0}$ holds. Otherwise, that is, if $x_{0}^{i} \in \mathcal{X}_{c}$ holds for all $i \in\left\{1,2, \ldots, N_{N}\right\}$, it determines that $\Sigma$ is $(T, \mathcal{X})$-p-controllable-w- $\left(\varepsilon_{N}, \delta_{N}\right)$ (line 5).

Algorithm $\mathrm{N}$ terminates after at most $N_{N}$ times checking the condition $x_{0}^{i} \notin \mathcal{X}_{c}$. It is also noticed that the negative result ( $\Sigma$ is $(T, \mathcal{X})$-uncontrollable) is guaranteed in the strict sense; thus, algorithms $\mathrm{P}$ and $\mathrm{N}$ are complementary to each other.

Remark 4: Since i.i.d. random vectors are generated on $\mathcal{X}_{0}$, under the situation that $\mathcal{X}_{0}-\mathcal{X}_{c}$ is a nonempty set of measure zero in $\mathcal{R}^{n}$, algorithm $\mathrm{N}$ cannot determine that $\Sigma$ is $(T, \mathcal{X})$-uncontrollable, i.e., $\mathcal{X}_{0}-\mathcal{X}_{c}$ is a nonempty set, even though $N_{N}$ is given as $N_{N}:=\infty$. This is a theoretical limitation of the method based on Lemma 10. However, we can obtain at least some information on the $(T, \mathcal{X})$-controllability in the sense of (31) even for such a case.

\section{Probabilistic Controllability Analysis With Two-Sided Error}

Based on algorithm $\mathrm{P}$ (except for subalgorithm 1) and algorithm N, we propose here the two-sided error algorithm (algorithm $\mathrm{T}$ ), which can determine if or not the system is $(T, \mathcal{X})$-controllable with a probabilistic accuracy; so the random sampling of both the mode sequence $\mathbb{\in} \in \mathcal{I}^{T-1}$ and the initial state $x_{0} \in \mathcal{X}_{0}$ is considered. First, we obtain the following result from Lemmas 3 and 10.

Lemma 11: For the system $\Sigma$, suppose that $T \in\{2,3, \ldots\}$ and $\mathcal{X} \subseteq \mathcal{R}^{n(T+1)}$ are given, and assume A3) and A4). For given $\varepsilon_{P} \in(0,1), \delta_{P} \in(0,1), \varepsilon_{N} \in(0,1)$, and $\delta_{N} \in(0,1)$, let $N_{P}$ and $N_{N}$ be the numbers defined by (13) and (30). If the following holds:

C4) for all i.i.d. random vectors $x_{0}^{i}\left(i=1,2, \ldots, N_{N}\right)$ from the uniform distribution on $\mathcal{X}_{0}$, some $\rrbracket^{j_{*}} \in \mathcal{I}^{T-1}$ of $N_{P}$ i.i.d. random vectors $\rrbracket^{j}\left(j=1,2, \ldots, N_{P}\right)$ from the uniform distribution on $\mathcal{I}^{T-1}$ satisfies $x_{0}^{i} \in \mathcal{X}_{c}^{\jmath^{j *}}$

then, (31) holds. Otherwise, there exists an $x_{0} \in \mathcal{X}_{0}$ satisfying (14). 
From (8), if C4) holds, then C3) holds, and if not, then C2) holds. Thus, checking $\mathrm{C} 4)$ can determine if $\Sigma$ is $(T, \mathcal{X})$-p-controllable-w- $\left(\varepsilon_{N}, \delta_{N}\right)$ or $(T, \mathcal{X})$-p-uncontrollable-w- $\left(\varepsilon_{P}, \delta_{P}\right)$. Thus, under A3) and A4), the following two-sided error algorithm is presented.

\section{Algorithm T: Two-Sided Error Algorithm}

0: Given $T \in\{2,3, \ldots\}, \mathcal{X} \subseteq \mathcal{R}^{n(T+1)}, \varepsilon_{P} \in(0,1)$, $\delta_{P} \in(0,1), \varepsilon_{N} \in(0,1)$, and $\delta_{N} \in(0,1)$;

1: Let $N_{P}$ and $N_{N}$ be the numbers defined by (13) and (30), respectively;

2: Generate i.i.d. random vectors $x_{0}^{1}, x_{0}^{2}, \ldots, x_{0}^{N_{N}}$ from the uniform distribution on $\mathcal{X}_{0}$;

3: $i:=1$;

4: Generate i.i.d. random vectors $\rrbracket^{1}, \rrbracket^{2}, \ldots, \rrbracket^{N_{P}}$ from the uniform distribution on $\mathcal{I}^{T-1}$;

5: If $x_{0}^{i} \notin \mathcal{X}_{c}^{\mathbb{\natural}^{j}}$ for all $j \in\left\{1,2, \ldots, N_{P}\right\}$ [i.e., C4) does not hold], then Halt: return " $(T, \mathcal{X})$-p-uncontrollable-w- $\left(\varepsilon_{P}, \delta_{P}\right)$ ";

6: If $i==N_{N}$ then Halt: return

"( $(T, \mathcal{X})$-p-controllable-w- $\left(\varepsilon_{N}, \delta_{N}\right)$ ";

$7: i:=i+1$, go to line 4 .

Algorithm T terminates after at most $N_{P} N_{N}$ times checking $x_{0}^{i} \notin \mathcal{X}_{c}^{\rrbracket^{j}}$, i.e., solving $\operatorname{LP} 3\left(I_{0}^{i}, x_{0}^{i}, \rrbracket^{j}\right)$ for $I_{0}^{i} \in \mathcal{I}$ satisfying $x_{0}^{i} \in \mathcal{S}_{I_{0}^{i}}$.

\section{Complexity Analysis of Three Algorithms}

This section discusses the computational complexities of algorithms $\mathrm{P}, \mathrm{N}$, and $\mathrm{T}$. For simplicity of discussion, in addition to A2)-A4), the following holds:

\section{A1') $\mathcal{X}$ is a polyhedron}

which implies A1) is assumed. This allows us to express $\mathcal{X}$ by some linear inequalities, and then, the geometrical complexity of $\mathcal{X}$ can be easily related to the computational complexities of the algorithms.

Let $N_{\mathcal{X}} \in \mathcal{N}$ be the number of the inequalities characterizing $\mathcal{X}$, i.e., $N_{\mathcal{X}}$ is the sum of the row sizes of the matrices $\Gamma$ and $\hat{\Gamma}$ for $\mathcal{X}$ expressed as the form

$$
\begin{aligned}
& \mathcal{X}=\left\{\left[\begin{array}{lll}
x_{0}^{\top} & \mathbb{X}^{\top} & x_{T}^{\top}
\end{array}\right]^{\top}\right.
\end{aligned}
$$

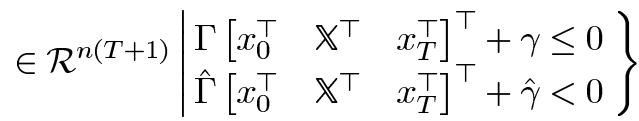

where $\gamma$ and $\hat{\gamma}$ are some vectors and let $N_{\mathcal{S}}:=\max _{I \in \mathcal{I}} p_{I}+\hat{p}_{I}$, where $p_{I}$ and $\hat{p}_{I}$ are defined for (2). Then, we will estimate the computational complexities of the proposed algorithms with respect to the variables of the $(T, \mathcal{X})$-controllability problem and the variables of the algorithms, i.e., the specification variables $T$ and $N_{\mathcal{X}}$ of the $(T, \mathcal{X})$-controllability, the scale/complexity variables $n, M$, and $N_{\mathcal{S}}$ of the system $\Sigma$, and the accuracy variables $\varepsilon_{P}, \delta_{P}, \varepsilon_{N}$, and $\delta_{N}$. Note that $N_{\mathcal{X}}$ expresses the complexity of the form of $\mathcal{X}$. Then the following result is obtained.

Lemma 12: For $T \in\{2,3, \ldots\}$ and $\mathcal{X} \subseteq \mathcal{R}^{n(T+1)}$ satisfying A1') and A2)-A4), the following statements hold.

1) For fixed $n$, algorithm $P$ is a polynomial-time algorithm with respect to $T, N_{\mathcal{X}}, M, N_{\mathcal{S}}, \varepsilon_{P}$, and $\delta_{P}$.

2) For fixed $T$, algorithm $\mathrm{N}$ is a polynomial-time algorithm with respect to $N_{\mathcal{X}}, n, M, N_{\mathcal{S}}, \varepsilon_{N}$, and $\delta_{N}$.

3) Algorithm $T$ is a polynomial-time algorithm with respect to $T, N_{\mathcal{X}}, n, M, N_{\mathcal{S}}, \varepsilon_{P}, \delta_{P}, \varepsilon_{N}$, and $\delta_{N}$ (i.e., to all variables).

Proof:

1) It is proven by the fact from Lemma 8 that algorithm $\mathrm{P}$ terminates after solving at most $M N_{P}\left(1+N_{0}+\right.$ $\left.\sum_{i=0}^{N_{F}} N_{w}(i)+N_{F}\right)$ LP problems and at most $M N_{P}(1+$ $N_{F}$ ) convex hull problems.

First, $N_{P}$ in (13) is bounded by a polynomial function of $\varepsilon_{P}$ and $\delta_{P}$, and $N_{0}$ is an arbitrarily given constant. Second, as for $N_{w}(i)$ and $N_{F}$, it turns out that for fixed $n$, they are bounded by a polynomial function of $\operatorname{card}\left(\operatorname{vert}\left(\overline{\mathcal{X}}_{c}^{I_{0} \rrbracket}\right)\right)$. Noting that $\overline{\mathcal{X}}_{c}^{I_{0} \rrbracket}$ can be characterized by at most $N_{\mathcal{T}_{c}^{I_{0}} \text { घ }}$ inequalities (see the proof of Lemma 2), where $N_{\mathcal{T}_{f}^{I_{0}}}$ is the number of the inequalities characterizing $\mathcal{T}_{c}^{\mathcal{I}_{0}}$ in (9), it follows from the well-known result on the number of vertices of a polyhedron (e.g., [40]) that

$$
\begin{aligned}
\operatorname{card}\left(\operatorname{vert}\left(\overline{\mathcal{X}}_{c}^{I_{0} \rrbracket}\right)\right) \leq & \left(\begin{array}{c}
\left.N_{\mathcal{T}_{c}^{I_{0} \rrbracket}}-\left\lfloor\frac{n+1}{2}\right\rfloor\right) \\
N_{\mathcal{T}_{c}^{I_{0} \rrbracket}}-n
\end{array}\right) \\
& +\left(\begin{array}{c}
\left.N_{\mathcal{T}_{c}^{I_{0} \rrbracket}}-\left\lfloor\frac{n+2}{2}\right\rfloor\right) . \\
N_{\mathcal{T}_{c}^{I_{0} \rrbracket}}-n
\end{array}\right) .
\end{aligned}
$$

Then, by definition, $N_{\mathcal{T}_{c}^{I_{0} \rrbracket}}$ is bounded by a polynomial function of $T, N_{\mathcal{X}}, n$, and $N_{\mathcal{S}}$. Hence, for fixed $n, N_{w}(i)$ and $N_{F}$ are bounded by a polynomial function of $N_{\mathcal{X}}$, $T$, and $N_{\mathcal{S}}$.

Third, in a series of LP problems $\left[\operatorname{LP} 1\left(I_{0}, \mathbb{}\right)\right.$ in (16), $\operatorname{LP} 2(w)$ in (17), and LP3 $\left(I_{0}, x_{0}, \mathbb{}, \mathbb{\text { in }}(22)\right]$, the dimension of the variables and the number of the constraints are bounded by a polynomial function of $T, N_{\mathcal{X}}, n$, and $N_{\mathcal{S}}$; thus, the LP problems can be solved by a polynomial-time algorithm (e.g., the interior point method) with respect to $T, N_{\mathcal{X}}, n$, and $N_{\mathcal{S}}$.

Finally, for fixed $n$, the convex hull problem can be solved by a polynomial-time algorithm with respect to $N_{w}(i)$ and $N_{F}$, i.e., to the variables $T, N_{\mathcal{X}}$, and $N_{\mathcal{S}}$. In fact, for $r:=N_{w}(i)+\operatorname{card}\left(\operatorname{vert}\left(\tilde{\mathcal{X}}_{c}^{I_{0}}(i)\right)\right)$, the problem corresponds to finding the convex hull of $r$ points in $\mathcal{R}^{n}$, and then, there exists a polynomial-time algorithm with respect to $r$ for determining the hyperplane representation and $\operatorname{vert}\left(\tilde{\mathcal{X}}_{c}^{I_{0} \rrbracket}(i)\right)$ can be computed in a polynomial time with respect to $N_{F}$ (from the fact $\left.\operatorname{card}\left(\operatorname{vert}\left(\tilde{\mathcal{X}}_{c}^{I_{0} \rrbracket}(i)\right)\right) \leq N_{F}\right)$. Note that the convex hull computation needs an exponential time with respect to $n$. 
TABLE II

Relation OF THREe Probabilistic APPROACHES

\begin{tabular}{|c|c|c|c|c|}
\hline & & Algorithm P & Algorithm N & Algorithm T \\
\hline \multicolumn{2}{|c|}{$\overline{\text { Random variables in algorithm }}$} & $\overline{\mathbb{I}}$ & $\bar{x}_{0}$ & $\bar{I}$ and $x_{0}$ \\
\hline \multirow{2}{*}{$\begin{array}{l}\text { Solution of } \\
\text { algorithm }\end{array}$} & Positive result & $(T, \mathcal{X})$-controllable & $\begin{array}{c}(T, \mathcal{X}) \text {-p-controllable } \\
\left.\text {-w-( } \varepsilon_{N}, \delta_{N}\right)\end{array}$ & $\begin{array}{c}(T, \mathcal{X}) \text {-p-controllable } \\
\text {-w- }\left(\varepsilon_{N}, \delta_{N}\right)\end{array}$ \\
\hline & Negative result & $\begin{array}{c}(T, \mathcal{X}) \text {-p-uncontrollable } \\
\text {-w- }\left(\varepsilon_{P}, \delta_{P}\right)\end{array}$ & $(T, \mathcal{X})$-uncontrollable & $\begin{array}{c}(T, \mathcal{X}) \text {-p-uncontrollable } \\
\text {-w- }\left(\varepsilon_{P}, \delta_{P}\right)\end{array}$ \\
\hline \multirow{3}{*}{$\begin{array}{l}\text { Computation amount } \\
\text { in the worst case }\end{array}$} & w.r.t. $T$ & Polynomial & Exponential & Polynomial \\
\hline & w.r.t. $n$ & Exponential & Polynomial & Polynomial \\
\hline & w.r.t. the other variables & Polynomial & Polynomial & Polynomial \\
\hline
\end{tabular}

Hence, the statement 1) holds.

2) and 3) The conditions of line 4 in algorithm $\mathrm{N}$ and line 5 in algorithm $\mathrm{T}$ can be checked by solving $\operatorname{LP} 3\left(I_{0}, x_{0}, \mathbb{},\right)$ for every $\llbracket \in \mathcal{I}^{T-1}$ and for every $\llbracket \in\left\{\rrbracket^{1}, \rrbracket^{2}, \ldots, \rrbracket^{N_{P}}\right\}$, respectively. Thus, algorithms $\mathrm{N}$ and $\mathrm{T}$ terminate after solving at most $N_{N} M^{T-1}$ LP problems and at most $N_{N} N_{P}$ LP problems, respectively. Then, since $N_{N}$ in (30) is bounded by a polynomial function of $\varepsilon_{N}$ and $\delta_{N}$, 2 ) and 3) are proven in a similar way to 1 ).

It is remarked that the proof of Lemma 12 also shows that subalgorithm 1 has a polynomial-time complexity with respect to $T, N_{\mathcal{X}}, M$, and $N_{\mathcal{S}}$, and an exponential-time complexity with respect to $n$.

Table II condenses the features and the computational complexities of algorithms $\mathrm{P}, \mathrm{N}$, and $\mathrm{T}$. Note that they are complementary to each other for their computational complexities as well as the results determined by them.

\section{FURTHER IMPROVEMENTS OF THREE ALGORITHMS}

This section presents several techniques to execute the previously described algorithms with a smaller size of samples than $N_{P}$ in (13) and $N_{N}$ in (30). Note here that Table II still holds even when such techniques are used.

First, we improve algorithm P. For $T \in\{3,4, \ldots\}$, let

$$
\tilde{\mathcal{I}}^{T-1}:=\left\{\llbracket \in \mathcal{I}^{T-1} \mid \xi_{\rrbracket_{(k)} \rrbracket_{(k+1)}}=1, k=1,2, \ldots, T-2\right\}
$$

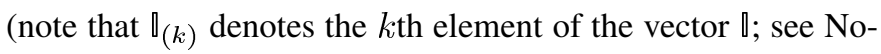
tation in Section I), and let $N_{\Xi}$ be the sum of all elements of $\Xi^{T-2}$, where $\xi_{I J}(I, J \in \mathcal{I})$ and $\Xi$ are given by

$\xi_{I J}:= \begin{cases}1, & \text { if } \exists(x, u) \in \mathcal{S}_{I} \times \mathcal{R}^{m} \text { s.t. } A_{I} x+B_{I} u+a_{I} \in \mathcal{S}_{J} \\ 0, & \text { otherwise }\end{cases}$ $\Xi:=\left[\begin{array}{cccc}\xi_{00} & \xi_{01} & \ldots & \xi_{0(M-1)} \\ \xi_{10} & \xi_{11} & \ldots & \xi_{1(M-1)} \\ \vdots & \vdots & \ddots & \vdots \\ \xi_{(M-1) 0} & \xi_{(M-1) 1} & \ldots & \xi_{(M-1)(M-1)}\end{array}\right]$.

Since $\xi_{I J}=1$ expresses that the discrete transition from $I(k)=$ $I$ to $I(k+1)=J$ is feasible via some control input $u(k) \in \mathcal{R}^{m}$, $\Xi$ denotes the adjacency matrix of the directed graph as shown in Fig. 3, where each node corresponds to each $I \in \mathcal{I}$ and the edge from the node $I$ to the node $J$ corresponds to the possible discrete transition from $I$ to $J$ in one time step, e.g., a time step from some $k$ to $k+1$. Thus, $\tilde{\mathcal{I}}^{T-1}$ expresses the set of the mode

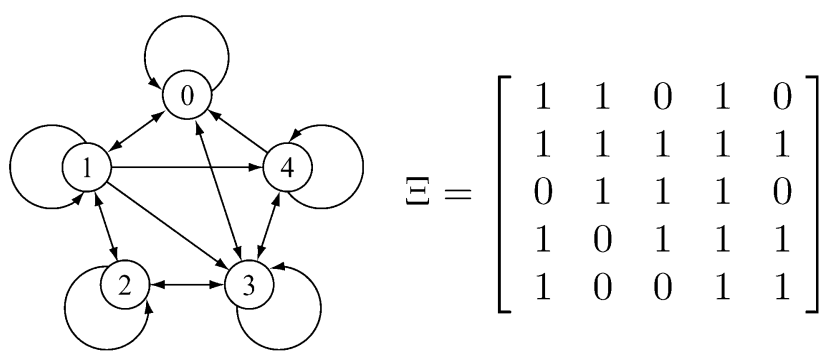

Fig. 3. Example of possible discrete transitions expressed by directed graph and adjacency matrix.

sequences which are the directed paths of length $T-2$ in the graph, and $N_{\Xi}$ denotes the cardinality of $\tilde{\mathcal{I}}^{T-1}$. Note that the relation $\mathcal{I}_{c}^{T-1}\left(x_{0}\right) \subseteq \tilde{\mathcal{I}}^{T-1}$ holds for every $x_{0} \in \mathcal{X}_{0}$. Then, the following result improves algorithm $\mathrm{P}$ by using the specified subset $\tilde{\mathcal{I}}^{T-1}$.

Lemma 13: For the system $\Sigma$, suppose that $T \in\{3,4, \ldots\}$, $\mathcal{X} \subseteq \mathcal{R}^{n(T+1)}$, and $x_{0} \in \mathcal{X}_{0}$ are given. For given $\varepsilon_{P} \in(0,1)$ satisfying $\varepsilon_{P} \operatorname{card}\left(\mathcal{I}^{T-1}\right) / N_{\Xi}<1$ and $\delta_{P} \in(0,1)$, let

$$
\tilde{N}_{P}:=\left\lceil\frac{\ln \frac{1}{\delta_{P}}}{\ln \frac{1}{1-\tilde{\varepsilon}_{P}}}\right\rceil
$$

where $\tilde{\varepsilon}_{P}:=\varepsilon_{P} \operatorname{card}\left(\mathcal{I}^{T-1}\right) / N_{\Xi}$. Then, if for all $\tilde{N}_{P}$ i.i.d. random vectors $\rrbracket^{i}\left(i=1,2, \ldots, \tilde{N}_{P}\right)$ from the uniform distribution on $\tilde{\mathcal{I}}^{T-1}, \rrbracket^{i} \notin \mathcal{I}_{c}^{T-1}\left(x_{0}\right)$ holds [see (10) for the definition of $\left.\mathcal{I}_{c}^{T-1}\left(x_{0}\right)\right]$, the relation (14) holds.

Proof: In a similar way to Lemma 3, if for all i.i.d. random vectors $\rrbracket^{i}\left(i=1,2, \ldots, \tilde{N}_{P}\right)$ from the uniform distribution on $\tilde{\mathcal{I}}^{T-1}$, ${ }^{i} \notin \mathcal{I}_{c}^{T-1}\left(x_{0}\right)$ holds, then the relation $\operatorname{Prob}\left\{\operatorname{card}\left(\mathcal{I}_{c}^{T-1}\left(x_{0}\right)\right) / \operatorname{card}\left(\tilde{\mathcal{I}}^{T-1}\right) \leq \tilde{\varepsilon}_{P}\right\} \geq 1-\delta_{P}$ holds. This implies (14).

Lemma 13 implies that for $T \in\{3,4, \ldots\}$, the number of samples required to verify (14) is less than or equal to the number given by (13) because of $\varepsilon_{P} \leq \tilde{\varepsilon}_{P}$. Thus, we can obtain a more efficient algorithm than algorithm $\mathrm{P}$, where the statements in lines 0 and 1 of algorithm $\mathrm{P}$ are replaced by the following.

0: Given $T \in\{3,4, \ldots\}, \mathcal{X} \subseteq \mathcal{R}^{n(T+1)}, \varepsilon_{P} \in(0,1)$ satisfying $\varepsilon_{P} \operatorname{card}\left(\mathcal{I}^{T-1}\right) / N_{\Xi}<1$, and $\delta_{P} \in(0,1)$;

1: Let $\tilde{N}_{P}$ be the number defined by (35);

and $\mathcal{I}^{T-1}$ in line 2 and $N_{P}$ in lines 2 and 7 are replaced by $\tilde{\mathcal{I}}^{T-1}$ and $\tilde{N}_{P}$, respectively. Note that $\Xi$ can be easily obtained; 
in fact, for given $I, J \in \mathcal{I}, \xi_{I J}=1$ holds if and only if the LP problem

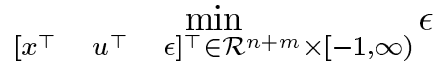

$$
\begin{aligned}
& \text { subject to }\left\{\begin{array}{l}
C_{I} x+d_{I} \leq 0 \\
\hat{C}_{I} x+\hat{d}_{I} \leq \epsilon \mathbf{1} \\
C_{J}\left(A_{I} x+B_{I} u+a_{I}\right)+d_{J} \leq 0 \\
\hat{C}_{J}\left(A_{I} x+B_{I} u+a_{I}\right)+\hat{d}_{J} \leq \epsilon \mathbf{1}
\end{array}\right.
\end{aligned}
$$

is feasible and its optimal value, denoted by $\epsilon^{*}$, is negative, where $\epsilon$ is a scalar variable.

Furthermore, a significant issue on line 2 in the aforementioned new algorithm, i.e., the uniform sampling method on $\tilde{\mathcal{I}}^{T-1}$, is discussed. As a method to generate i.i.d. random vectors from the uniform distribution on a set, the rejection method, ${ }^{1}$ for example, is well known. However, similarly to the case of [41], in the new algorithm, this method based on the sampling on $\mathcal{I}^{T-1}$ is inefficient when the difference between $\operatorname{card}\left(\mathcal{I}^{T-1}\right)$ and $\operatorname{card}\left(\tilde{\mathcal{I}}^{T-1}\right)$ is large, because a huge number of i.i.d. random vectors on $\mathcal{I}^{T-1}$ will have to be generated to obtain an i.i.d. random vector on $\tilde{\mathcal{I}}^{T-1}$. Hence, an efficient sampling method in this case is proposed as follows. This generates an i.i.d. random vector from the uniform distribution

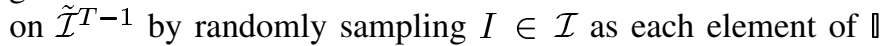
according to a probability distribution specified based on the number of the directed paths from $I$ on the directed graph.

Lemma 14: For the system $\Sigma$, suppose that $T \in\{3,4, \ldots\}$ is given. For $I, J \in \mathcal{I}$ and $k \in\{1,2, \ldots, T-1\}$, let $\Xi_{I J}^{k-1}$ be the $(I+1, J+1)$ th element of $\Xi^{k-1}$ and let $\mathcal{I}_{I}:=\left\{J \in \mathcal{I} \mid \Xi_{I J}=\right.$ $1\}$. Let also $\mathbb{\rrbracket}_{(k)} \in \mathcal{I}(k=1,2, \ldots, T-1)$ be a random value on $\mathcal{I}$ with the probability distribution

$$
\operatorname{Prob}\left\{\rrbracket_{(1)}=I\right\}:=\frac{\sum_{J \in \mathcal{I}} \Xi_{I J}^{T-2}}{N_{\Xi}}
$$

and

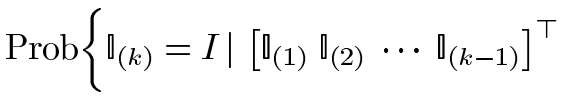

$$
\begin{aligned}
& \left.=\left[\begin{array}{llll}
\tilde{\mathbb{I}}_{(1)} \tilde{\mathbb{I}}_{(2)} & \cdots & \tilde{\mathbb{}}_{(k-1)}
\end{array}\right]^{\top} \in \tilde{\mathcal{I}}^{k-1}\right\} \\
& := \begin{cases}0, & \text { if } I \notin \mathcal{I}_{\tilde{\mathbb{D}}_{(k-1)}} \\
\frac{\sum_{J \in \mathcal{I}} \Xi_{I J}^{T-1-k}}{\sum_{J \in \mathcal{I}} \Xi_{\tilde{\mathbb{I}}_{(k-1)} J}^{T-k}}, & \text { if } I \in \mathcal{I}_{\tilde{\mathbb{D}}_{(k-1)}}\end{cases}
\end{aligned}
$$

for $k=2,3, \ldots, T-1$. Then, the relation $\operatorname{Prob}\{\mathbb{\square}=\tilde{\mathbb{\natural}}\}=1 / N_{\Xi}$ holds for every $\tilde{\mathbb{}} \in \tilde{\mathcal{I}}^{T-1}$.

Proof: Since $\mathcal{I}_{I}$ expresses the set of $J \in \mathcal{I}$ such that the discrete transition from $I(k)=I$ to $I(k+1)=J$ is possible, the relation $\tilde{\mathbb{I}}_{(k)} \in \mathcal{I}_{\tilde{\mathbb{I}}_{(k-1)}}$ holds for every $\tilde{\mathbb{}} \in \tilde{\mathcal{I}}^{T-1}$ and $k \in$ $\{2,3, \ldots, T-1\}$. Thus, from the fact that $\sum_{J \in \mathcal{I}} \Xi_{I J}^{0}=1$ holds for every $I \in \mathcal{I}$, the relation

\footnotetext{
${ }^{1}$ Rejection method: Let $\mathcal{Z}$ and $\overline{\mathcal{Z}}$ be sets $(\mathcal{Z} \subset \overline{\mathcal{Z}})$ such that we cannot easily generate an i.i.d. random vector from the uniform distribution on $\mathcal{Z}$ but we can generate it on $\overline{\mathcal{Z}}$. Then, an i.i.d. random vector from the uniform distribution on $\mathcal{Z}$ is generated by the two steps: 1) generate an i.i.d. random vector $z$ from the uniform distribution on $\overline{\mathcal{Z}}$ and 2) if $z \in \mathcal{Z}, z$ is accepted, otherwise, $z$ is rejected and 1) is executed again.
}

$$
\begin{aligned}
& \operatorname{Prob}\{\llbracket=\tilde{\mathbb{1}}\}
\end{aligned}
$$

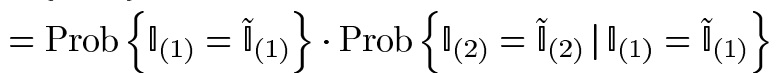

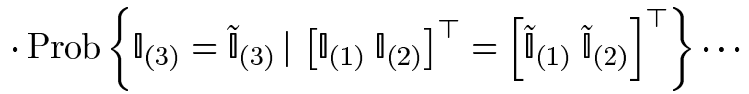

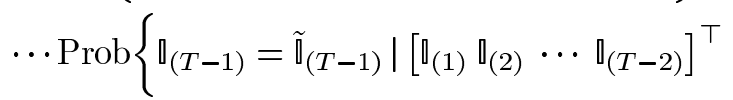

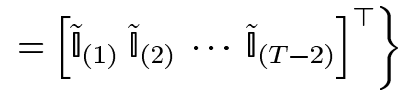

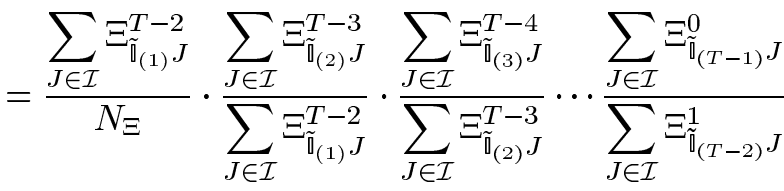

$$
\begin{aligned}
& =\frac{1}{N_{\Xi}}
\end{aligned}
$$

holds for every $\tilde{\mathbb{}} \in \tilde{\mathcal{I}}^{T-1}$.

Lemma 14 implies that the random sampling on $\mathcal{I}^{T-1}$ according to the probability distribution of (36) and (37) gives us a random vector from the uniform distribution on $\tilde{\mathcal{I}}^{T-1}$. Thus, instead of the rejection method, we can use the method based on Lemma 14 as an efficient technique.

Remark 5: There must exist other techniques for efficiently executing algorithm P. For example, it may be useful to exploit the enumeration technique of the feasible mode sequences in [7].

Next, in a similar way to Lemma 13, we obtain the following result for algorithm N. It improves the algorithm by removing $x_{0} \in \mathcal{X}_{0}$ obviously satisfying $x_{0} \in \mathcal{X}_{c}$ from the sampled set $\mathcal{X}_{0}$.

Lemma 15: For the system $\Sigma$, suppose that $T \in\{2,3, \ldots\}$ and $\mathcal{X} \subseteq \mathcal{R}^{n(T+1)}$ are given. Let $\mathcal{I}_{\text {nipt }}:=\left\{I \in \mathcal{I} \mid \operatorname{rank} B_{I}=\right.$ $n\}$ and assume the following:

A5) $\mathcal{I}_{\text {nipt }} \neq \emptyset, \mathcal{X}_{T} \neq \emptyset$, and there exists an $\rrbracket \in \mathcal{I}_{\text {nipt }}^{T-1}$ satisfying $\mathcal{S}_{\emptyset} \cap \mathcal{X}_{i m}^{T-1} \neq \emptyset$.

For $\tilde{\mathcal{X}}_{0}:=\mathcal{X}_{0}-\bigcup_{I_{0} \in \mathcal{I}_{\text {nipt }}} \mathcal{S}_{I_{0}}$, and given $\varepsilon_{N} \in(0,1)$ satisfying $\varepsilon_{N} \operatorname{vol}\left(\mathcal{X}_{0}\right) / \operatorname{vol}\left(\tilde{\mathcal{X}}_{0}\right)<1$ and $\delta_{N} \in(0,1)$, let also

$$
\tilde{N}_{N}:=\left\lceil\frac{\ln \frac{1}{\delta_{N}}}{\ln \frac{1}{1-\tilde{\varepsilon}_{N}}}\right\rceil
$$

where $\tilde{\varepsilon}_{N}:=\varepsilon_{N} \operatorname{vol}\left(\mathcal{X}_{0}\right) / \operatorname{vol}\left(\tilde{\mathcal{X}}_{0}\right)$. Then, if for all $\tilde{N}_{N}$ i.i.d. random vectors $x_{0}^{i}\left(i=1,2, \ldots, N_{N}\right)$ from the uniform distribution on $\tilde{\mathcal{X}}_{0}, x_{0}^{i} \in \mathcal{X}_{c}$ holds, the relation (31) holds.

Proof: In 2) of Lemma 1, since $B_{\bar{I}}^{\perp}$ is given as, for example, $B_{I}^{\perp}=0_{n \times 1}$ for every $I \in \mathcal{I}_{\text {nipt }}$, the relations $E_{I_{0} 0}^{a}=0$ and $E_{I_{0} \rrbracket}^{b}=0$ hold for every $\left(I_{0}, \mathbb{}\right) \in \mathcal{I}_{\text {nipt }}^{T}$. Then, it follows from (6) that for given $I_{0} \in \mathcal{I}_{\text {nipt }}$ and $\rrbracket \in \mathcal{I}_{\text {nipt }}^{T-1}$ satisfying $\mathcal{S}_{\emptyset} \cap \mathcal{X}_{i m}^{T-1} \neq \emptyset, \mathcal{X}_{c}^{I_{0} \rrbracket}$ is given as $\mathcal{X}_{c}^{I_{0} \rrbracket}=\mathcal{S}_{I_{0}} \cap \mathcal{X}_{0}$ under A5); so $\bigcup_{I_{0} \in \mathcal{I}_{\text {nipt }}} \mathcal{S}_{I_{0}} \cap \mathcal{X}_{0} \subseteq \mathcal{X}_{c}$ holds from (7) and (8). Therefore, if for all i.i.d. random vectors $x_{0}^{i}\left(i=1,2, \ldots, \tilde{N}_{N}\right)$ from the uniform distribution on $\tilde{\mathcal{X}}_{0}, x_{0}^{i} \in \mathcal{X}_{c}$ holds, then the relation $\operatorname{Prob}\left\{\operatorname{vol}\left(\mathcal{X}_{0}-\mathcal{X}_{c}\right) / \operatorname{vol}\left(\tilde{\mathcal{X}}_{0}\right) \leq \tilde{\varepsilon}_{N}\right\} \geq 1-\delta_{N}$, i.e., (31), holds. 
In Lemma 15, the number of samples given by (38), which is required to verify (31), is less than or equal to the number given by (30). Thus, we can obtain a more efficient algorithm than algorithm $\mathrm{N}$, where the statements in lines 0 and 1 of algorithm $\mathrm{N}$ are replaced by the following:

0 : Given $T \in\{2,3, \ldots\}, \mathcal{X} \subseteq \mathcal{R}^{n(T+1)}, \varepsilon_{N} \in(0,1)$ satisfying $\varepsilon_{N} \operatorname{vol}\left(\mathcal{X}_{0}\right) / \operatorname{vol}\left(\tilde{\mathcal{X}}_{0}\right)<1$, and $\delta_{N} \in(0,1)$;

1: Let $\tilde{N}_{N}$ be the number defined by (38);

and $\mathcal{X}_{0}$ in line 2 and $N_{N}$ in lines 2 and 5 are replaced by $\tilde{\mathcal{X}}_{0}$ and $\tilde{N}_{N}$, respectively.

Lemmas 13-15 are also useful for obtaining a more efficient algorithm than algorithm $\mathrm{T}$, where the statements in lines 0 and 1 of algorithm $\mathrm{T}$ are replaced by the following:

0: Given $T \in\{3,4, \ldots\}, \mathcal{X} \subseteq \mathcal{R}^{n(T+1)}, \varepsilon_{P} \in(0,1)$ satisfying $\varepsilon_{P} \operatorname{card}\left(\mathcal{I}^{T-1}\right) / N_{\Xi}<1, \delta_{P} \in(0,1), \varepsilon_{N} \in(0,1)$ satisfying $\varepsilon_{N} \operatorname{vol}\left(\mathcal{X}_{0}\right) / \operatorname{vol}\left(\tilde{\mathcal{X}}_{0}\right)<1$, and $\delta_{N} \in(0,1)$;

1: Let $\tilde{N}_{P}$ and $\tilde{N}_{N}$ be the numbers defined by (35) and (38), respectively;

and $\mathcal{X}_{0}$ in line $2, \mathcal{I}^{T-1}$ in line $4, N_{P}$ in lines 4 and 5 , and $N_{N}$ in lines 2 and 6 , are replaced by $\tilde{\mathcal{X}}_{0}, \tilde{\mathcal{I}}^{T-1}, \tilde{N}_{P}$, and $\tilde{N}_{N}$, respectively.

\section{EXAMPLES}

Consider the system $\Sigma$ in Example 1, where $T:=10$ and $\mathcal{X}$ is given in the same way. For these $T$ and $\mathcal{X}$, assumptions A1)-A4) hold, and so algorithms $\mathrm{P}, \mathrm{N}$, and $\mathrm{T}$ can be applied. In addition, since A5) holds, Lemma 15 can be also applied.

We apply here the improved versions of algorithms $\mathrm{P}, \mathrm{N}$, and $\mathrm{T}$ to the $(T, \mathcal{X})$-controllability problem. We set $\varepsilon_{P}:=10^{-3}$ and $\delta_{P}:=10^{-3}$, so $\tilde{N}_{P}=719$ by (35), and set $\varepsilon_{N}:=10^{-3}$ and $\delta_{N}:=10^{-3}$, so $\tilde{N}_{N}=5178$ by (38) [note that $N_{P}=$ $N_{N}=6905$ from (13) and (30)]. Under such a situation, for example, algorithm $\mathrm{P}$ can determine if $\Sigma$ is $(T, \mathcal{X})$-controllable or $(T, \mathcal{X})$-p-uncontrollable-w- $\left(10^{-3}, 10^{-3}\right)$, i.e., there exists an $x_{0} \in \mathcal{X}_{0}$ satisfying $\operatorname{Prob}\left\{\square \in \mathcal{I}_{c}^{T-1}\left(x_{0}\right)\right\} \leq 0.1 \%$ with probability greater than or equal to $99.9 \%$. In addition, it turns out that $\Xi$ for every $\zeta \in\{1,2\}$ is given by Fig. 3 , and $\tilde{\mathcal{X}}_{0}$ in Lemma 15 is given as $\tilde{\mathcal{X}}_{0}=\mathcal{X}_{0}-\mathcal{S}_{1}$ (note $\mathcal{I}_{\text {nipt }}=\{1\}$ ).

The numerical results of the probabilistic controllability analysis are shown in Table III, where the equality condition of line 6 in algorithm $P$ is checked by [10], and $\tilde{\mathcal{X}}_{c}^{I_{0} \rrbracket}(0)$ in subalgorithm 1 is defined as (26). The symbols " $Y$," " $N$," and "?" are defined in the same way to Example 1, while the symbol " $Y^{*}$ " implies that $\Sigma$ is $(T, \mathcal{X})$-p-controllable-w- $\left(\varepsilon_{N}, \delta_{N}\right)$ and " $N^{*}$ " implies that $\Sigma$ is $(T, \mathcal{X})$-p-uncontrollable-w- $\left(\varepsilon_{P}, \delta_{P}\right)$. The results in Table III are based on ten trials, where the algorithms answered the same result in every trial, and the computation time and the number of the LP problems express the maximum value, the mean, and the minimum value in the trials. This table shows that the $(T, \mathcal{X})$-controllability problem, which may not be solved in a deterministic way as shown in Example 1, can be solved within a practically short time by the proposed approach.
TABLE III

Results of Probabilistic Controllability ANALYsis For $T:=10$. (a) $\operatorname{CASE} \zeta=1$. (b) $\operatorname{CASE} \zeta=2$

\begin{tabular}{|c|c||c|c|c|}
\hline \multicolumn{2}{|c||}{ (a) } \\
\hline \hline \multicolumn{2}{|c||}{ Algorithm } & $\mathrm{P}$ & $\mathrm{N}$ & $\mathrm{T}$ \\
\hline \multicolumn{2}{|c|}{$(T, \mathcal{X})$-controllability } & $\mathrm{N}^{*}$ & $\mathrm{~N}$ & $\mathrm{~N}^{*}$ \\
\hline Computation time & max. & 133.2 & $1,600.4$ & 7.9 \\
[sec] & mean & 121.7 & $1,404.4$ & 7.2 \\
& min. & 114.4 & $1,274.0$ & 6.2 \\
\hline Number of & max. & 4,200 & 98,964 & 534 \\
LP problems solved & mean & 4,117 & 83,631 & 473 \\
[times] & min. & 3,906 & 73,394 & 395 \\
\hline
\end{tabular}

(b)

\begin{tabular}{|c|c||c|c|c|}
\hline \multicolumn{2}{|c||}{ Algorithm } & $\mathrm{P}$ & $\mathrm{N}$ & $\mathrm{T}$ \\
\hline \hline \multicolumn{2}{|c||}{$(T, \mathcal{X})$-controllability } & $\mathrm{Y}$ & $\mathrm{Y}^{*}$ & $\mathrm{Y}^{*}$ \\
\hline Computation time & max. & 5.2 & 154.8 & 156.7 \\
[sec] & mean & 3.9 & 124.7 & 136.1 \\
\hline Number of & min. & 1.9 & 102.0 & 121.3 \\
\hline LP problems solved & mean & 271 & 10,584 & 10,729 \\
[times] & min. & 105 & 8,439 & 9,318 \\
\hline
\end{tabular}

$\mathrm{Y}:(T, \mathcal{X})$-controllable, $\quad \mathrm{Y}^{*}:(T, \mathcal{X})$-p-controllable-w- $\left(\varepsilon_{N}, \delta_{N}\right)$, $\mathrm{N}:(T, \mathcal{X})$-uncontrollable, $\mathrm{N}^{*}:(T, \mathcal{X})$-p-uncontrollable-w- $\left(\varepsilon_{P}, \delta_{P}\right)$.

TABLE IV

Results of PROBABILISTIC CONTROLLABILITY ANALYSIS FOR $T:=50$. (a) $\operatorname{CASE} \zeta=1$. (b) $\operatorname{CASE} \zeta=2$

\begin{tabular}{|c|c||c|c|c|}
\multicolumn{5}{|c||}{ (a) } \\
\hline \multicolumn{2}{|c||}{ Algorithm } & $\mathrm{P}$ & $\mathrm{N}$ & $\mathrm{T}$ \\
\hline \hline \multicolumn{2}{|c|}{$(T, \mathcal{X})$-controllability } & $\mathrm{N}^{*}$ & $?$ & $\mathrm{~N}^{*}$ \\
\hline Computation time & max. & 2785.1 & & 564.5 \\
{$[$ [sec] } & mean & 2690.0 & - & 515.3 \\
& min. & 2639.2 & & 444.2 \\
\hline Number of & max. & 42,292 & & 5,963 \\
LP problems solved & mean & 41,209 & - & 5,345 \\
[times] & min. & 40,905 & & 4,456 \\
\hline
\end{tabular}

(b)

\begin{tabular}{|c|c|c|c|c|}
\hline \multicolumn{2}{|l|}{ Algorithm } & $\mathrm{P}$ & $\mathbf{N}$ & $\mathrm{T}$ \\
\hline \multicolumn{2}{|c|}{$\overline{(T, \mathcal{X}) \text {-controllability }}$} & $\mathrm{Y}$ & $\overline{\mathrm{Y}^{*}}$ & $\overline{\mathrm{Y}^{*}}$ \\
\hline \multirow{3}{*}{$\begin{array}{l}\text { Computation time } \\
\text { [sec] }\end{array}$} & $\max$. & 12.2 & 1227.7 & 1101.9 \\
\hline & mean & 9.7 & 1057.7 & 896.5 \\
\hline & $\min$. & 6.7 & 851.1 & 762.4 \\
\hline \multirow{3}{*}{$\begin{array}{c}\text { Number of } \\
\text { LP problems solved } \\
\text { [times] }\end{array}$} & max. & 250 & 11,850 & 10,522 \\
\hline & mean & 208 & 10,287 & 9,131 \\
\hline & $\min$. & 150 & 8,845 & 8,192 \\
\hline
\end{tabular}

On the other hand, Table IV shows the numerical results for $T:=50$, where by considering that $\operatorname{card}\left(\mathcal{I}^{T-1}\right)$ is huge, that is, $5^{49}$ in this case, we set $\varepsilon_{P}$ to be relatively small, i.e., $\varepsilon_{P}:=$ $10^{-10}$ and $\mathcal{X}, \delta_{P}, \varepsilon_{N}$, and $\delta_{N}$ are given in the same way as the case $T:=10$. Here, algorithms $\mathrm{P}$ and $\mathrm{T}$ can solve the problem for both $\zeta=1$ and $\zeta=2$ in a practically short time. Meanwhile, algorithm $\mathrm{N}$ cannot solve it in the case of $\zeta=1$. This is because the number of LP problems to be solved exponentially increases with the size of $T$ in algorithm N. However, since three algorithms are complementary to each other for computational complexity as shown in Table II, we can get some probabilistic information on the controllability from algorithms $\mathrm{P}$ and $\mathrm{T}$.

\section{CONCLUSION}

This paper has proposed a probabilistic approach to the controllability analysis of the discrete-time PWA systems. Based on a characterization of controllability, three kinds of polynomialtime algorithms have been proposed for approximately solving the problem in a probabilistic way, where the random sampling of the mode sequence and/or the initial state is used. It has been 
also shown that the proposed algorithms are practically useful for examples to which the deterministic methods will not be able to be applied. The proposed probabilistic approach can be straightforwardly extended to the case of the sampled-data PWA systems, and also to the reachability problem and the observability problem [42], [38], [43]. We hope that the proposed framework will be a practical tool for understanding system properties of complex dynamical systems such as biosystems (e.g., gene regulatory network [22], [37]). Such a topic is one of future works.

\section{APPENDIX}

\section{PROOFS OF THE RESULTS IN SECTION III.A.2}

\section{A. Proof of Lemma 4}

Since $\mathcal{X}_{c}^{I_{0} \rrbracket}$ is a polyhedron obtained by the projection of $\mathcal{T}_{c}^{I_{0} \rrbracket}$ onto the $x_{0}$-space, $\mathcal{X}_{c}^{I_{0} \rrbracket} \neq \emptyset$ if and only if $\mathcal{T}_{c}^{I_{0} \rrbracket} \neq \emptyset$. On the other hand, it follows from (9) that $\mathcal{T}_{c}^{I_{0} \rrbracket} \neq \emptyset$ holds if and only if $\operatorname{LP} 1\left(I_{0}, \mathbb{\square}\right)$ is feasible and $\epsilon_{1}^{*}<0$. These facts prove that 1$)$ is equivalent to 2 ).

\section{B. Proof of Lemma 5}

Since from the proof of Lemma 2, the relation

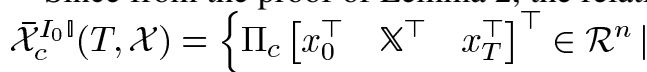

$$
\begin{aligned}
& \left.\left[\begin{array}{lll}
x_{0}^{\top} & \mathbb{X}^{\top} & x_{T}^{\top}
\end{array}\right]^{\top} \in \overline{\mathcal{T}}_{c}^{I_{0} \rrbracket}(T, \mathcal{X})\right\}
\end{aligned}
$$

holds for $\Pi_{c}:=\left[\begin{array}{lll}\boldsymbol{I}_{n} & 0_{n \times n(T-1)} & 0_{n \times n}\end{array}\right]$, we have

$$
\begin{aligned}
& \min _{x_{0} \in \overline{\mathcal{X}}_{c}^{I_{0}}} w x_{0} \\
& =\min _{x_{0} \in\left\{\Pi_{c}\left[x_{0}^{\top} \quad \mathbb{X}^{\top} \quad x_{T}^{\top}\right]^{\top} \in \mathcal{R}^{n} \mid\left[\begin{array}{lll}
x_{0}^{\top} & \mathbb{X}^{\top} & x_{T}^{\top}
\end{array}\right]^{\top} \in \overline{\mathcal{T}}_{c}^{I_{0} 0}\right\}} w x_{0}
\end{aligned}
$$

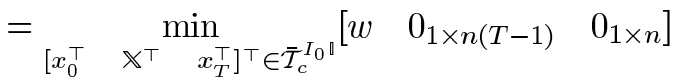

$$
\begin{aligned}
& \times\left[\begin{array}{lll}
x_{0}^{\top} & \mathbb{X}^{\top} & x_{T}^{\top}
\end{array}\right]^{\top}
\end{aligned}
$$

which is $\operatorname{LP} 2(w)$. Thus, $x_{0}^{*}(w)$ is a boundary point of $\overline{\mathcal{X}}_{c}^{I_{0}}$.

Note here that it follows from (40) that there exists an optimal solution $\left(x_{0}^{*}(w), \mathbb{X}^{*}(w), x_{T}^{*}(w) w\right)$ to $\operatorname{LP} 2(w)$, because $\overline{\mathcal{X}}_{c}^{I_{0} \rrbracket}$ is a bounded (and closed) polyhedron from assumptions A1) and A2), and $\overline{\mathcal{T}}_{c}^{I_{0} \rrbracket} \neq \emptyset$ holds from 1) of Lemma 4 .

\section{Proof of Lemma 6}

1) Since, from (40), $x_{0}^{*}\left(-w_{i}\right)$ is an optimal solution to the LP problem $\min _{x_{0} \in \overline{\mathcal{X}}_{c}^{I_{0}} \rrbracket}-w_{i} x_{0}$ under A1), A2), and 1) of Lemma $4,-w_{i} x_{0}^{*}\left(-w_{i}\right) \leq-w_{i} x_{0}$ holds for every $x_{0} \in$ $\overline{\mathcal{X}}_{c}^{I_{0} \rrbracket}$. This implies that $w_{i} x_{0}^{*}\left(-w_{i}\right)+v_{i} \leq 0$ holds (that is, $v_{i} \leq-w_{i} x_{0}^{*}\left(-w_{i}\right)$ ) if and only if $w_{i} x_{0}+v_{i} \leq 0$ for every $x_{0} \in \overline{\mathcal{X}}_{c}^{I_{0} \rrbracket}$. Thus, b) holds if and only if $x_{0} \in \tilde{\mathcal{X}}_{c}^{I_{0} \rrbracket}$ holds for every $x_{0} \in \overline{\mathcal{X}}_{c}^{I_{0}}$. On the other hand, it follows from the relation $\tilde{\mathcal{X}}_{c}^{I_{0} \rrbracket} \subseteq \overline{\mathcal{X}}_{c}^{I_{0} \rrbracket}$ that a) holds if and only if $x_{0} \in \tilde{\mathcal{X}}_{c}^{I_{0} \rrbracket}$ holds for every $x_{0} \in \overline{\mathcal{X}}_{c}^{I_{0}}$. Therefore, a) is equivalent to b).

2) This follows from the fact that $x_{0}^{*}\left(-w_{i}\right) \in \overline{\mathcal{X}}_{c}^{I_{0} \rrbracket}$ and $x_{0}^{*}\left(-w_{i}\right) \notin \tilde{\mathcal{X}}_{c}^{I_{0} \rrbracket}$ hold for $i \in\left\{1,2, \ldots, N_{w}\right\}$ such that $w_{i} x_{0}^{*}\left(-w_{i}\right)+v_{i} \leq 0$ does not hold.

\section{Proof of Lemma 7}

Under A1), A2), and 1) of Lemma 4, there exist some nonempty faces of $\overline{\mathcal{X}}_{c}^{I_{0}}$. Then, we first prove that one and only one of the following two relations holds for each $\left.i \in\left\{1,2, \ldots, N_{F}\right\}: 1\right) \operatorname{ri}\left(\mathcal{F}_{i}\right) \subseteq \mathcal{X}_{c}^{I_{0} \rrbracket}$ and 2) $\mathcal{X}_{c}^{I_{0} \rrbracket} \cap \operatorname{ri}\left(\mathcal{F}_{i}\right)=\emptyset$.

For each $i \in\left\{1,2, \ldots, N_{F}\right\}$, there exists a hyperplane $\left\{x_{0} \in\right.$ $\left.\mathcal{R}^{n} \mid G_{i} x_{0}+g_{i}=0\right\}$ satisfying $\overline{\mathcal{X}}_{c}^{I_{0} \rrbracket} \subseteq\left\{x_{0} \in \mathcal{R}^{n} \mid G_{i} x_{0}+g_{i} \leq\right.$ $0\}$ and $\mathcal{F}_{i}=\overline{\mathcal{X}}_{c}^{I_{0}} \cap\left\{x_{0} \in \mathcal{R}^{n} \mid G_{i} x_{0}+g_{i}=0\right\}(\neq \emptyset)$, where $G_{i}$ and $g_{i}$ are a row vector and a scalar, respectively; so if for given $\left.i \in\left\{1,2, \ldots, N_{F}\right\}, 1\right)$ does not hold, $\mathcal{X}_{c}^{I_{0} \rrbracket} \subseteq \overline{\mathcal{X}}_{c}^{I_{0}} \cap\left\{x_{0} \in\right.$ $\left.\mathcal{R}^{n} \mid G_{i} x_{0}+g_{i}<0\right\}$ holds (because $\mathcal{X}_{c}^{I_{0}}$ can be characterized by some inequalities), namely

$$
\mathcal{X}_{c}^{I_{0}} \cap\left\{x_{0} \in \mathcal{R}^{n} \mid G_{i} x_{0}+g_{i}=0\right\}=\emptyset
$$

holds. Then, from the relation $\mathcal{F}_{i} \subseteq\left\{x_{0} \in \mathcal{R}^{n} \mid G_{i} x_{0}+g_{i}=\right.$ $0\}$, it follows that $\mathcal{X}_{c}^{I_{0} \rrbracket} \cap\left\{x_{0} \in \mathcal{R}^{n} \mid G_{i} x_{0}+g_{i}=0\right\}=\emptyset$ implies 2). On the other hand, 2) obviously implies that 1) does not hold. Therefore, one and only one of the relations 1) or 2) holds for each $i \in\left\{1,2, \ldots, N_{F}\right\}$. From this fact, (21) is proven as follows. Since $\overline{\mathcal{X}}_{c}^{I_{0} \rrbracket}=\bigcup_{j \in\left\{1,2, \ldots, N_{F}\right\}} \operatorname{ri}\left(\mathcal{F}_{j}\right)$, the maximum subset of the boundary of $\overline{\mathcal{X}}_{c}^{I_{0} \rrbracket}$ that is not included in $\mathcal{X}_{c}^{I_{0}}$ is

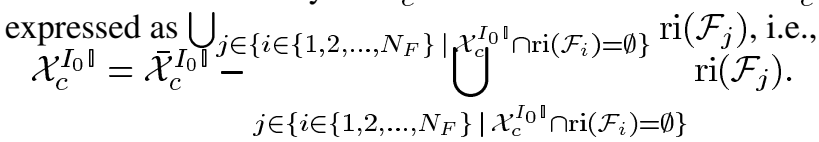

Therefore, since 2) and the condition $\mathcal{X}_{c}^{I_{0} \rrbracket} \cap \mathcal{F}_{i}=\emptyset$ are equivalent from the fact that (41) (i.e., $\mathcal{X}_{c}^{I_{0}} \cap \mathcal{F}_{i}=\emptyset$ ) holds if 1) does not hold, the right-hand side of (42) is equal to the right-hand side of (21), which completes the proof.

\section{E. Proof of (27)}

At first, the following result is prepared.

Lemma 16: For the system $\Sigma$, suppose that $T \in\{2,3, \ldots\}$, $\mathcal{X} \subseteq \mathcal{R}^{n(T+1)},\left(I_{0}, \mathbb{\square}\right) \in \mathcal{I}^{T}$, and a nonempty closed polyhedron $\tilde{\mathcal{X}}_{c}^{I_{0} \rrbracket}\left(\subset \overline{\mathcal{X}}_{c}^{I_{0}}\right)$ are given, and assume that A1), A2), and 1) of Lemma 4 hold. Suppose also that some $i \in\left\{1,2, \ldots, N_{w}\right\}$ satisfying $w_{i} x_{0}^{*}\left(-w_{i}\right)+v_{i}>0$ is given (note that $N_{w} \in \mathcal{N}_{+}$, $w_{i} \in \mathcal{R}^{1 \times n}$, and $v_{i} \in \mathcal{R}\left(i=1,2, \ldots, N_{w}\right)$ for $\tilde{\mathcal{X}}_{c}^{I_{0} \rrbracket}$ are defined in Lemma 6). Then, $x_{0}^{*}\left(-w_{i}\right) \notin \operatorname{ri}\left(\mathcal{F}_{j}\left(\overline{\mathcal{X}}_{c}^{I_{0}}\right)\right)$ holds for every $j \in\left\{1,2, \ldots, N_{F}\right\}$ satisfying $\tilde{\mathcal{X}}_{c}^{I_{0} \rrbracket} \cap \mathcal{F}_{j}\left(\overline{\mathcal{X}}_{c}^{I_{0} \rrbracket}\right) \neq \emptyset$.

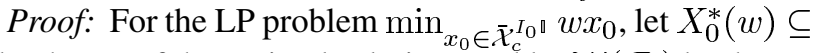
$\mathcal{R}^{n}$ be the set of the optimal solutions and let $\mathcal{W}\left(\mathcal{F}_{j}\right)$ be the set of all $w \in \mathcal{R}^{1 \times n}$ satisfying $\mathcal{F}_{j} \subseteq X_{0}^{*}(w)$. Then, since one and only one of the relations $\mathcal{F}_{j} \subseteq X_{0}^{*}(w)$ or $\operatorname{ri}\left(\mathcal{F}_{j}\right) \cap X_{0}^{*}(w)=\emptyset$ holds, if $w \in \mathcal{W}\left(\mathcal{F}_{j}\right)$, then $\mathcal{F}_{j} \subseteq X_{0}^{*}(w)$ holds; otherwise, $\operatorname{ri}\left(\mathcal{F}_{j}\right) \cap X_{0}^{*}(w)=\emptyset$ (that is, $\left.x_{0}^{*}(w) \notin \operatorname{ri}\left(\mathcal{F}_{j}\right)\right)$ holds. On the other hand, if $\tilde{\mathcal{X}}_{c}^{I_{0} \rrbracket} \cap \mathcal{F}_{j} \neq \emptyset$, then there exists an $x_{0} \in \mathcal{F}_{j}$ satisfying $w_{i} x_{0}+v_{i} \leq 0$ (i.e., $\left.-w_{i} x_{0}^{*}\left(-w_{i}\right)<-w_{i} x_{0}\right)$, namely, the relation $\mathcal{F}_{j} \subseteq X_{0}^{*}\left(-w_{i}\right)$ does not hold (note that this implies that $\operatorname{ri}\left(\mathcal{F}_{j}\right) \cap X_{0}^{*}\left(-w_{i}\right)=\emptyset$ holds); so $-w_{i} \notin \mathcal{W}\left(\mathcal{F}_{j}\right)$ holds for every $j \in\left\{1,2, \ldots, N_{F}\right\}$ satisfying $\tilde{\mathcal{X}}_{c}^{I_{0} \rrbracket} \cap \mathcal{F}_{j} \neq \emptyset$. Therefore, it follows that the relation $x_{0}^{*}\left(-w_{i}\right) \notin \operatorname{ri}\left(\mathcal{F}_{j}\right)$ holds for every $j \in\left\{1,2, \ldots, N_{F}\right\}$ satisfying $\tilde{\mathcal{X}}_{c}^{I_{0}} \cap \mathcal{F}_{j} \neq \emptyset$.

Lemma 16 implies that for every $i \in\left\{1,2, \ldots, N_{w}\right\}$ satisfying $w_{i} x_{0}^{*}\left(-w_{i}\right)+v_{i}>0, x_{0}^{*}\left(-w_{i}\right)$ is included in the set $\operatorname{ri}\left(\mathcal{F}_{j}\right)$ for a $j \in\left\{1,2, \ldots, N_{F}\right\}$ satisfying $\tilde{\mathcal{X}}_{c}^{I_{0} \rrbracket} \cap \mathcal{F}_{j}=\emptyset$; roughly speaking, if the condition $\overline{\mathcal{X}}_{c}^{I_{0} \rrbracket} \neq \tilde{\mathcal{X}}_{c}^{I_{0} \rrbracket}$ is verified by 
1) of Lemma $6, x_{0}^{*}\left(-w_{i}\right)$ is always a newly obtained boundary point of $\overline{\mathcal{X}}_{c}^{I_{0} \rrbracket}$ that is included in some face $\mathcal{F}_{j}$ satisfying $\tilde{\mathcal{X}}_{c}^{I_{0} \square} \cap$ $\mathcal{F}_{j}=\emptyset$. Thus, $\operatorname{card}(\mathcal{E}(i))<\operatorname{card}(\mathcal{E}(i+1))\left(i=0,1, \ldots, i_{f}-\right.$ 1) and $\operatorname{card}\left(\mathcal{E}\left(i_{f}\right)\right)=N_{F}$ hold, namely, (27) holds.

In Example 2, since $\mathcal{E}(0)=\{2,3,4,5,7,8,9,11\}$ and $\mathcal{E}(1)=\{1,2, \ldots, 11\}$, we have $\operatorname{card}(\mathcal{E}(0))<$ $\operatorname{card}(\mathcal{E}(1))=N_{F}$. In fact, $\tilde{\mathcal{X}}_{c}^{I_{0} \rrbracket}(0) \cap \operatorname{ri}\left(\mathcal{F}_{j}\right) \neq \emptyset$ holds for $j \in\{2,3,4,5,7,8,9,11\}$ and $\tilde{w}_{6} x_{0}^{*}\left(-\tilde{w}_{6}\right)+\tilde{v}_{6}>0$ hold; so $x_{0}^{*}\left(-\tilde{w}_{6}\right) \notin \operatorname{ri}\left(\mathcal{F}_{j}\right)$ for $j \in\{2,3,4,5,7,8,9,11\}$.

\section{ACKNOWLEDGMENT}

The authors would like to thank Prof. S. Hara, The University of Tokyo, Tokyo, Japan, and Y. Fujisaki, the Associate Professor at the Kobe University, Kobe, Japan, for valuable comments.

\section{REFERENCES}

[1] J. Ezzine and A. H. Haddad, "Controllability and observability of hybrid systems," Int. J. Control, vol. 49, no. 6, pp. 2045-2055, 1989.

[2] S. S. Ge, Z. Sun, and T. H. Lee, "Reachability and controllability of switched linear discrete-time systems," IEEE Trans. Autom. Control, vol. 46, no. 9, pp. 1437-1441, Sep. 2001.

[3] E. D. Sontag, "Interconnected automata and linear systems: A theoretical framework in discrete-time," in Lecture Notes in Computer Science, ser. 1066, R. Alur, T. A. Henzinger, and E. D. Sontag, Eds. Berlin, Germany: Springer-Verlag, 1996, pp. 436-448, Hybrid Systems III: Verification and Control.

[4] V. D. Blondel and J. N. Tsitsiklis, "Complexity of stability and controllability of elementary hybrid systems," Automatica, vol. 35, no. 3, pp. 479-489, 1999.

[5] A. Bemporad, G. Ferrari-Trecate, and M. Morari, "Observability and controllability of piecewise affine and hybrid systems," IEEE Trans. Autom. Control, vol. 45, no. 10, pp. 1864-1876, Oct. 2000.

[6] A. Bemporad and M. Morari, "Verification of hybrid systems via mathematical programming," in Lecture Notes in Computer Science, ser. 1569, F. W. Vaandrager and J. H. van Schuppen, Eds. Berlin, Germany: Springer-Verlag, 1999, pp. 31-45, Hybrid Systems: Computation and Control.

[7] A. Bemporad, F. D. Torrisi, and M. Morari, "Optimization-based verification and stability characterization of piecewise affine and hybrid systems," in Lecture Notes in Computer Science, ser. 1790, N. Lynch and B. H. Krogh, Eds. Berlin, Germany: Springer-Verlag, 2000, pp. 45-58, Hybrid Systems: Computation and Control.

[8] A. Bemporad, M. Morari, V. Dua, and E. N. Pistikopoulos, "The explicit linear quadratic regulator for constrained systems," Automatica, vol. 38, no. 1, pp. 3-20, 2002.

[9] F. Borrelli, Constrained Optimal Control of Linear and Hybrid Systems. Berlin, Germany: Springer-Verlag, 2003.

[10] M. Kvasnica, P. Grieder, M. Baotić, and F. J. Christophersen, "Multiparametric toolbox (MPT)," 2004 [Online]. Available: http://control.ee. ethz.ch/ mpt/

[11] S. Azuma and J. Imura, "Controllability of sampled-data piecewise affine systems," in Proc. IFAC Conf. Anal. Design Hybrid Syst., 2003, pp. $129-134$.

[12] J. Imura, "Optimal control of sampled-data piecewise affine systems," Automatica, vol. 40, no. 4, pp. 661-669, 2004.

[13] S. Azuma and J. Imura, "Synthesis of optimal controllers for piecewise affine systems with sampled-data switching," Automatica, vol. 42, no. 5, pp. 697-710, 2006.

[14] E. S. Lemch, S. Sastry, and P. E. Caines, "Global controllability of hybrid systems with controlled and autonomous switchings," in Lecture Notes in Computer Science, ser. 2034, M. D. Di Benedetto and A. L. Sangiovanni-Vincentelli, Eds. Berlin, Germany: Springer-Verlag, 2001, pp. 375-386, Hybrid Systems: Computation and Control.

[15] M. K. Camlibel, W. P. M. H. Heemels, and J. M. Schumacher, "On the controllability of bimodal piecewise linear systems," in Lecture Notes in Computer Science, ser. 2993, R. Alur and G. J. Pappas, Eds. Berlin, Germany: Springer-Verlag, 2004, pp. 250-264, Hybrid Systems: Computation and Control.
[16] J. H. van Schuppen, "A sufficient condition for controllability of a class of hybrid systems," in Lecture Notes in Computer Science, ser. 1386, T. A. Henzinger and S. Sastry, Eds. Berlin, Germany: Springer-Verlag, 1998, pp. 374-383, Hybrid Systems: Computation and Control.

[17] J. Lygeros, D. N. Godbole, and S. Sastry, "Verified hybrid controllers for automated vehicles," IEEE Trans. Autom. Control, vol. 43, no. 4, pp. 522-539, Apr. 1998.

[18] J. Lygeros, C. Tomlin, and S. Sastry, "Controllers for reachability specifications for hybrid systems," Automatica, vol. 35, no. 3, pp. 349-370, 1999.

[19] C. Tomlin, G. J. Pappas, and S. Sastry, "Conflict resolution for air traffic management: A study in multiagent hybrid systems," IEEE Trans. Autom. Control, vol. 43, no. 4, pp. 509-521, Apr. 1998.

[20] I. Mitchell and C. Tomlin, "Overapproximating reachable sets by Hamilton-Jacobi projections," J. Sci. Comput., vol. 19, no. 1, pp. 323-346, 2003.

[21] G. Lafferriere, G. J. Pappas, and S. Yovine, "Symbolic reachability computation for families of linear vector fields," J. Symb. Comput., vol. 32, no. 3, pp. 231-253, 2001.

[22] R. Ghosh, A. Tiwari, and C. Tomlin, "Automated symbolic reachability analysis; with application to Delta-Notch signaling automata," in Lecture Notes in Computer Science, ser. 1790, F. Wiedijk, O. Maler, and A. Pnueli, Eds. Berlin, Germany: Springer-Verlag, 2003, pp. 233-248, Hybrid Systems: Computation and Control.

[23] T. A. Henzinger, P. H. Ho, and H. Wong-Toi, "HyTech: A model checker for hybrid systems," Int. J. Soft. Tools Tech. Transfer, vol. 1, no. 1-2, pp. 110-122, 1997.

[24] A. Chutinan and B. H. Krogh, "Verification of polyhedral-invariant hybrid automata using polygonal flow pipe approximations," in Lecture Notes in Computer Science, ser. 1569, F. W. Vaandrager and J. H. van Schuppen, Eds. Berlin, Germany: Springer-Verlag, 1999, pp. 76-90, Hybrid Systems: Computation and Control.

[25] O. Botchkarev and S. Tripakis, "Verification of hybrid systems with linear differential inclusions using ellipsoidal approximations," in Lecture Notes in Computer Science, ser. 1790, N. Lynch and B. H. Krogh, Eds. Berlin, Germany: Springer-Verlag, 2000, pp. 74-88, Hybrid Systems: Computation and Control.

[26] B. T. Polyak and R. Tempo, "Probabilistic robust design with linear quadratic regulators," Syst. Control Lett., vol. 43, no. 5, pp. 343-353, 2001.

[27] H. Ishii, T. Basar, and R. Tempo, "Randomized algorithms for quadratic stability of quantized sampled-data systems," Automatica, vol. 40, no. 5, pp. 839-846, 2004.

[28] H. Ishii, T. Basar, and R. Tempo, "Synthesis of switching rules for switched linear systems through randomized algorithms," in Proc. 42nd IEEE Conf. Decision Control, 2003, pp. 4788-4793.

[29] M. Prandini, J. Hu, J. Lygeros, and S. Sastry, "A probabilistic approach to aircraft conflict detection," IEEE Trans. Intell. Transp. Syst., vol. 1, no. 4, pp. 199-220, Dec. 2000.

[30] S. Prajna, A. Jadbabaie, and G. J. Pappas, "Stochastic safety verification using barrier certificates," in Proc. 43rd IEEE Conf. Decision Control, 2004, pp. 929-934.

[31] R. Tempo, E. W. Bai, and F. Dabbene, "Probabilistic robustness analysis: Explicit bounds for the minimum number of samples," Syst. Control Lett., vol. 30, no. 5, pp. 237-242, 1997.

[32] P. Khargonekar and A. Tikku, "Randomized algorithms for robust control analysis and synthesis have polynomial complexity," in Proc. 35th IEEE Conf. Decision Control, 1996, pp. 3470-3475.

[33] M. Vidyasagar, "Randomized algorithms for robust controller synthesis using statistical theory," Automatica, vol. 37, no. 10, pp. 1515-1528, 2001.

[34] E. D. Sontag, "Nonlinear regulation: The piecewise linear approach," IEEE Trans. Autom. Control, vol. AC-26, no. 2, pp. 346-358, Apr. 1981.

[35] “The Geometric Bounding Toolbox, User's Manual \& Reference," S. M. Veres, Ed., SysBrain, Birmingham, U.K., 2001.

[36] D. Bertsimas and J. N. Tsitsiklis, Introduction to Linear Optimization. Belmont, MA: Athena Scientific, 1997.

[37] E. Yanagisawa, S. Azuma, and J. Imura, "Controllability analysis for gene regulation networks and its application to luminescence bacterium," in Proc. Algebraic Biol. 2005-Computer Algebra Biol., H. Anai and K. Horimoto, Eds., 2005, pp. 51-60.

[38] S. Azuma and J. Imura, "A probabilistic approach to controllability/ reachability analysis of hybrid systems," in Proc. 43rd IEEE Conf. Decision Control, 2004, pp. 485-490. 
[39] C. N. Jones, E. C. Kerrigan, and J. M. Maciejowski, "Equality set projection: A new algorithm for the projection of polytopes in halfspace representation,” Tech. Rep. CUED/F-INFENG/TR.463, 2004 [Online]. Available: http://www-control.eng.cam.ac.uk/ cnj22/research/projection.html

[40] V. Chvátal, Linear Programming. New York: W. H. Freeman, 1983.

[41] G. C. Calafiore, F. Dabbene, and R. Tempo, "Randomized algorithms for probabilistic robustness with real and complex structured uncertainty," IEEE Trans. Autom. Control, vol. 45, no. 12, pp. 2218-2235, Dec. 2000.

[42] S. Azuma and J. Imura, "Probabilistic controllability analysis of sampled-data/discrete-time piecewise affine systems," in Proc. Amer. Control Conf., 2004, pp. 2528-2533.

[43] S. Azuma and J. Imura, "Polynomial-time probabilistic observability analysis of sampled-data piecewise affine systems," Syst. Control Lett., vol. 56, no. 11-12, pp. 685-694, 2007.

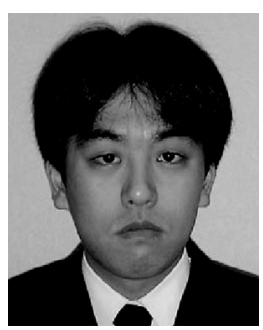

Shun-Ichi Azuma was born in Tokyo, Japan, in 1976. He received the B.Eng. degree in electrical engineering from Hiroshima University, Higashi Hiroshima, Japan, in 1999, and the M.Eng. and $\mathrm{Ph} . \mathrm{D}$. degrees in control engineering from Tokyo Institute of Technology, Tokyo, Japan, in 2001 and 2004, respectively.

He was a Research Fellow of the Japan Society for the Promotion of Science, Tokyo Institute of Technology, and a Visiting Scholar of Georgia Institute of Technology, Atlanta, from 2004 to 2005. Currently, he is an Assistant Professor at the Department of Systems Science, Graduate School of Informatics, Kyoto University, Kyoto, Japan. His research interests include control of hybrid systems.

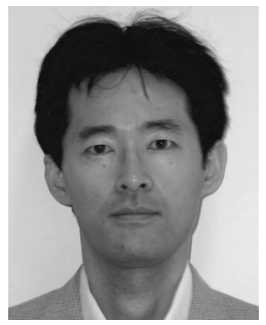

Jun-Ichi Imura was born in Gifu, Japan, in 1964. He received the M.S. degree in applied systems science and the Ph.D. degree in mechanical engineering from Kyoto University, Kyoto, Japan, in 1990 and 1995, respectively.

He served as a Research Associate at the Department of Mechanical Engineering, Kyoto University, from 1992 to 1996, and as an Associate Professor at the Division of Machine Design Engineering, Faculty of Engineering, Hiroshima University, Hiroshima, Japan, from 1996 to 2001. From May 1998 to April 1999, he was a Visiting Researcher at the Faculty of Mathematical Sciences, University of Twente, The Netherlands. Since 2001, he has been with the Department of Mechanical and Environmental Informatics, Graduate School of Information Science and Engineering, Tokyo Institute of Technology, Tokyo, Japan, where he is currently a Professor. His research interests include control of nonlinear systems and analysis and control of hybrid systems.

Dr. Imura has served as an Associate Editor for Asian Journal of Control and the International Journal of Modeling, Identification and Control. 